\title{
Potent Mixed Backbone Antisense Oligonucleotide Safety Suppressed Expression of Mutant C90RF72 Transcripts and Polypeptides: First in Human Pilot Study
}

Robert Brown ( $\sim$ Robert.Brown@umassmed.edu )

University of Massachusetts Medical School

Hélène Tran

Université de Lille, Inserm, CHU Lille, UMRS1172 Lille Neuroscience \& Cognition, F-59045 Lille, France

Michael Moazami

University of Massachusetts Medical School

Huiya Yang

University of Massachusetts Medical School

Diane McKenna-Yasek

University of Massachusetts Medical School

Catherine Douthwright

University of Massachusetts Medical School

Courtney PINTO

University of Massachusetts Medical School

Jake Metterville

University of Massachusetts Medical School

Minwook Shin

University of Massachusetts Medical School

Nitasha Sanil

University of Massachusetts Medical School

Craig Dooley

University of Massachusetts Medical School

Ajit Puri

University of Massachusetts Medical School

Alexandra Weiss

University of Massachusetts Medical School

Nichols Wightman

University of Massachusetts Medical School

Heather Gray-Edwards

University of Massachusetts Medical School 


\section{Miklos Marosfoi}

Lahey Clinic Medical Center

\section{Robert King}

University of Massachusetts Medical School https://orcid.org/0000-0002-5144-9110

\section{Thomas Kenderdine}

University of Connecticut

\section{Dan Fabris}

University of Connecticut

\section{Robert Bowser}

Barrow Neurological Institute

\section{Jonathan Watts}

University of Massachusetts Medical School https://orcid.org/0000-0001-5706-1734

\section{Article}

Keywords: amyotrophic lateral sclerosis (ALS), frontotemporal dementia (FTD)

Posted Date: February 12th, 2021

DOI: https://doi.org/10.21203/rs.3.rs-211236/v1

License: (c) (1) This work is licensed under a Creative Commons Attribution 4.0 International License. Read Full License

Version of Record: A version of this preprint was published at Nature Medicine on December 23rd, 2021. See the published version at https://doi.org/10.1038/s41591-021-01557-6. 


\section{POTENT MIXED BACKBONE ANTISENSE OLIGONUCLEOTIDE SAFELY SUPPRESSES EXPRESSION OF MUTANT C9ORF72 TRANSCRIPTS AND POLYDIPEPTIDES: FIRST IN HUMAN PILOT STUDY}

Hélène $\operatorname{Tran}^{1}$;, Michael P. Moazami ${ }^{2}$;, Huiya Yang ${ }^{1}$, Diane McKenna-Yasek ${ }^{1}$, Catherine Douthwright $^{1}$, Courtney Pinto ${ }^{1}$, Jake Metterville ${ }^{1}$, Minwook Shin $^{2}$, Nitasha Sanil ${ }^{3}$, Craig Dooley ${ }^{3}$, Ajit Puri ${ }^{4}$, Alexandra Weiss ${ }^{1}$, Nicholas Wightman ${ }^{1}$, Heather Gray-Edwards ${ }^{4}$, Miklos Marosfoi ${ }^{4}$, Robert M. King ${ }^{4,5}$, Thomas Kenderdine ${ }^{6}$, Daniele Fabris ${ }^{6}$, Robert Bowser ${ }^{7}$, Jonathan K. Watts ${ }^{2 *}$, Robert H. Brown, Jr ${ }^{1 *}$

\footnotetext{
Affiliations:

${ }^{1}$ Department of Neurology, University of Massachusetts Medical School, Worcester, MA, 01655 USA

${ }^{2}$ RNA Therapeutics Institute, University of Massachusetts Medical School, Worcester, MA, 01655 USA

${ }^{3}$ Research Pharmacy, University of Massachusetts Medical School, Worcester, MA, 01655 USA

${ }^{4}$ Department of Radiology, University of Massachusetts Medical School, Worcester, MA, 01655 USA

${ }^{5}$ Department of Biomedical Engineering, Worcester Polytechnic Institute, Worcester, MA, 01609, USA;

${ }^{6}$ Department of Chemistry, University of Connecticut, Storrs, CT, 06269, USA

${ }^{7}$ Departments of Neurology and Neurobiology, Barrow Neurological Institute, Phoenix, AZ, 85013, USA

*To whom correspondence should be addressed: robert.brown@umassmed.edu, jonathan.watts@umassmed.edu

$\$$ These authors contributed equally to the work
} 


\begin{abstract}
Words
Expansions of a $\mathrm{G}_{4} \mathrm{C}_{2}$ repeat in the C9ORF72 gene are the most common genetic cause of amyotrophic lateral sclerosis (ALS) and frontotemporal dementia (FTD), two devastating adultonset neurodegenerative disorders. Proposed disease mechanisms include a gain of toxic functions of the $\mathrm{G}_{4} \mathrm{C}_{2}$ repeats, implying that selective reduction in levels of the repeat-containing transcripts would represent a treatment strategy for this disorder. In the present study, using C9-ALS/FTD patient derived cells and C9ORF72 BAC transgenic mice, we have generated and optimized antisense oligonucleotides (ASOs) that selectively blunt expression of $\mathrm{G}_{4} \mathrm{C}_{2}$ repeat containing transcripts in both the sense and anti-sense strands of C9ORF72 and effectively suppress tissue levels of polyGP dipeptides. In a single patient harboring mutant $C 9 O R F 72$ with the $\mathrm{G}_{4} \mathrm{C}_{2}$ repeat expressions, repeated dosing by intrathecal delivery of the optimal ASO was well tolerated, leading to significant reductions in levels of CSF polyGP.
\end{abstract}

\title{
LIST OF ABBREVIATIONS
}

ALS: Amyotrophic Lateral Sclerosis; ASO: Antisense Oligonucleotide; C9BAC: Chromosome 9 Bacterial Artificial Chromosome; C9ORF72: Chromosome 9 Open Reading Frame; DPR: dipeptide repeat; FTD: Frontotemporal Dementia; HRE: hexanucleotide repeat expansion; ICV: Intracerebroventricular; LNA: Locked Nucleic Acid; MOE, 2'-O-MOE: 2'-O-2-methoxyethyl; MTD: Maximum Tolerated Dose; nmol: nanomole; PO: Phosphodiester; PS: Phosphorothioate; V1, V2, V3: transcript variants $1,2,3$

\section{INTRODUCTION 422 words}

A GGGGCC $\left(\mathrm{G}_{4} \mathrm{C}_{2}\right)$ hexanucleotide repeat expansion (HRE) in the first intron of the C9ORF72 (C9) gene is the most common genetic cause of amyotrophic lateral sclerosis (ALS) and frontotemporal dementia (FTD), two devastating adult-onset neurodegenerative disorders ${ }^{1-3}$. Proposed disease mechanisms include a partial loss of the C9ORF72 protein function (C9ORF72 haploinsufficiency) and acquired toxicity of the repeat expansion ${ }^{4-6}$. Transcription of C9ORF72 gene generates three transcript variants, V1, V2 and V3 (1) (Fig. 1a). V1 is translated to produce a short protein isoform (222 amino acids) while V2 and V3 generate the most predominant C9ORF72 protein (481 amino acids), which functions in vesicular trafficking ${ }^{7}$. Located adjacent 
to the promoter region of the most abundant $\mathrm{V} 2$ transcript variant, the $\mathrm{G}_{4} \mathrm{C}_{2}$ repeat expansion impairs its transcription, leading to C9ORF72 protein haploinsufficiency ${ }^{8-10}$, impaired function of myeloid cells ${ }^{11,12}$ and diminished neuronal viability ${ }^{13}$. Both sense and antisense transcripts encompassing the HRE in V1 and V3 generate RNA foci and undergo translation into atypical, aggregation-prone dipeptide repeat (DPR) proteins in all open reading frames ${ }^{14-17}$. These unusual DPRs are toxic in several experimental model systems ${ }^{18-23}$ Despite important advances in elucidating the molecular pathology of the expanded hexanucleotide repeats, there are no meaningful therapies for C9ORF72-related ALS or FTD ${ }^{24,25}$.

Antisense oligonucleotides (ASOs) can drive therapeutic effects by mechanisms that include splice-modulation ${ }^{26}$ or, if the ASO contains DNA, activation of endogenous RNase H ${ }^{27,28}$ to degrade the target RNA. The broad bioavailability of ASOs in the CNS, including both neurons and glial cells ${ }^{29}$ has prompted development of ASOs as therapy for dominantly transmitted genetic disorders of the central nervous system (e.g. Huntington's disease and ALS caused by mutations in the SOD1 gene) ${ }^{30-33}$.

Here, we report development of antisense oligonucleotides targeting the sense and antisense strands of C9ORF72 to treat ALS and FTD caused by the HRE in the V1 and V3 isoforms. Using different $\mathrm{C} 9$ related model systems, including patient-derived samples and two C9BAC transgenic mouse models ${ }^{34,35}$, we have generated ASOs that specifically reduce levels of the transcripts harboring the HRE as well as their DPR products, with minimal effects on the most abundant V2 isoform which does not contain the HRE. We show that modification of a subset of the phosphodiester internucleoside linkages significantly improves the tolerability without impairing the potency of the tested ASOs. We demonstrate that in a single patient harboring mutant C9ORF72 with the $\mathrm{G}_{4} \mathrm{C}_{2}$ repeat expressions, repeated intrathecal dosing of the optimal ASO was well tolerated and led to significant reductions in levels of CSF polyGP.

RESULTS 2708

$\mathrm{G}_{4} \mathrm{C}_{2}$ targeting ASO reduces the $\mathrm{C} 9 \mathrm{ORF} 72$ repeat containing transcripts in patient derived fibroblasts and C9BAC mouse derived neurons 
Because haploinsufficiency of C9ORF72 is thought to be adverse, we developed ASOs that target only the 5 'end of transcripts $\mathrm{V} 1$ and $\mathrm{V} 3$ that bear the $\mathrm{G}_{4} \mathrm{C}_{2}$ repeat expansion, sparing transcript $\mathrm{V} 2$. As it is not fully clear whether the repeat containing intron is retained or spliced out, we focused our effort on ASO sequences targeting the intron-repeat junction (Fig. 1a). Others have previously tested ASOs against this target region in patient derived samples in vitro with success ${ }^{36,37}$.

We developed a dual luciferase screen for ASOs that suppress expression of transcripts V1, V2 and V3 (Fig. S1a), and used this assay to narrow our focus to five ASOs. All ASOs were designed as gapmers to elicit RNase H-mediated target degradation, and all linkages were fully phosphorothioate (PS) modified. Sugars of the 5 ' and 3 ' regions of the ASOs were modified with either locked nucleic acid (LNA) or 2'-O-methoxyethyl (MOE) substitutions (Fig. 1b). All cytosine residues were 5-methylated to reduce immunogenicity ${ }^{38}$.

We treated primary C9-ALS/FTD patient-derived fibroblasts (with $>1000$ repeats, Fig. S1b, and showing visible nuclear C9ORF72 foci, Fig. S1c) with ASOs 1-5 at a dose of $100 \mathrm{nM}$ by lipid transfection. After 72 hours, we assayed the level of V1-V3 repeat containing transcripts by qRTPCR. Relative to untreated cells (UT) or a non-targeting control (NTC), all five ASOs reduced V1V3 expression to almost undetectable levels (Fig. 1c). Silencing was dose-dependent in patientderived fibroblasts (Fig. S1d) and in HEK293 cells expressing the dual luciferase reporter (Fig. S1e).

A hallmark of C9-ALS/FTD is the presence of repeat-containing RNA foci 1,2,17,36,39-41. Three days after ASO treatment, we used FISH to show that the number of cells with foci was markedly reduced from $80 \%$ in the untreated condition to $20-40 \%$ in the treated conditions. Moreover, fewer foci per cell were detected, showing that all five ASOs were potent inhibitors of $\mathrm{G}_{4} \mathrm{C}_{2}$ RNA foci (Fig. 1d-e).

Each of the five ASOs was also active by gymnotic (lipid-free) delivery to neurons. Primary cortical neurons were derived from E15.5 C9BAC embryos and treated with $1 \mu \mathrm{M}$ ASO at 5 days in vitro (DIV). 15 days after treatment (20DIV), expression of human V1V3 repeat containing transcript was significantly reduced (from $40 \%$ reduction with ASO1 to $80 \%$ with ASO3) in all treated conditions as compared to the non-targeting control or untreated condition (Fig. 1f).

From these in vitro experiments, we conclude that in various cell models (HEK 293 cells expressing a C9 intron1 reporter assay, C9-ALS/FTD patient-derived fibroblasts, and C9BAC 
mouse-derived cortical neurons), ASOs 1-5 (Fig. 1b) all potently inhibit expression of V1 and V3 repeat containing transcripts while sparing V2, and all are efficiently taken up by neurons without the need for a transfection reagent.

ASOs selectively reduce the C9ORF72 repeat-containing transcripts and peptides after CNS infusion in C9BAC mice

We next evaluated the properties of these ASOs in vivo in wild-type (WT) and C9ORF72 transgenic mice. ASOs can be delivered to the brain tissue and spinal cord through the surrounding cerebral spinal fluid (CSF) via an intracerebroventricular (ICV) bolus injection or osmotic pump infusion ${ }^{42}$. We first assessed ASO tolerability in wild-type C57BL/6 mice. Each animal received a single ICV bolus dose of one of our five ASOs. Each treatment group consisted of 2-4 mice. While these WT mice tolerated $30 \mathrm{nmol}$ of MOE-modified ASOs 4-5, more than 5 nmol of LNAmodified ASOs 1-3 were lethal. Mice injected with ASOs 1, 2 and 4 had severe seizure-like phenotypes upon recovery from anesthesia or did not survive 24 hours post-injection, while mice treated with ASOs 3 and 5 (that share the same nucleotide sequence but differ by their sugar modification) remained alert and responsive to stimuli with no obvious detrimental effects up to one week after injection (Fig. 2a).

Encouraged by the safety profile of ASOs 3 and 5 in WT mice, we then compared their tolerability and efficacy in C9BAC transgenic mice via ICV administration. C9BAC transgenic mice generated in our laboratory express approximately $600 \mathrm{G}_{4} \mathrm{C}_{2}$ repeat motifs within a truncated human C9ORF72 gene (from exons 1-6). Although these mice do not develop a motor phenotype, they fully recapitulate the distinct disease hallmarks including repeat containing RNA foci and DPR $^{42}$ and thus are a suitable C9-ALS/FTD mouse model for assessing efficacy of ASOs 3 and 5 activities in vivo. In our C9BAC mice, we were not able to safely perform intracerebroventricular infusions with more than $10 \mathrm{nmol}$ of any LNA-modified compounds using bolus injections. To overcome this limitation, we next used osmotic pumps, which allowed infusion of a higher total amount of LNA modified ASO (ASO3) and permitted comparison of its potency with the MOEmodified version (ASO5). Doses ranging from 2.5 to $20 \mathrm{nmol}$ per day of each ASO were continuously infused over 10 days into the right lateral ventricle of age-matched heterozygous C9BAC mice through a cannula using an implanted Alzet osmotic pump (Fig. 2b). Five to seven animals were used per group condition and animals infused with PBS or a non-targeting ASO were used as controls. Brains of animals sacrificed two weeks after the 10-day infusion demonstrated 
widespread ASO distribution throughout the brain with associated neuronal uptake (Fig. 2c). The cortex and spinal regions of animals treated with ASO3 and ASO5 demonstrated potent, dosedependent reduction in V1 and V3 repeat-containing transcripts in both the cortex and spinal cord regions (Fig. 2d, f) as compared to PBS-infused animals; no such reduction was seen in the nontargeting control animals. Importantly, despite their impact on V1 and V3, neither ASO3 nor ASO5 produced any substantial reduction of the level of the V2 transcript (and hence the total C9ORF72 transcript variants) (Fig. 2e, g). Poly-GP DPR was also reduced in the cortex of mice treated with both ASO3 and 5 (Fig. 2h).

With chronic infusion, the LNA and MOE-modified ASOs produced no adverse behavioral side effects throughout the course of ASO administration; all animals remained healthy until they were sacrificed at 21 days. Routine clinical blood chemistry and liver and kidney morphology after H\&E staining revealed no gross abnormalities (data not shown). Body weight monitoring from treatment onset to the time of sacrifice revealed a loss of up to $10 \%$ as compared to initial body weight before treatment; this was likely related to the pump implantation surgery as it also occurred in mice infused with PBS (Fig. 2i). At the $100 \mathrm{nmol}$ dose, mice receiving ASO3 showed more weight loss $(18 \%)$. We therefore focused on ASO5 for further optimization.

\section{Minimizing the PS content of ASO5 improves its safety profile without affecting its biological activity after a single CNS administration in C9BAC mice}

When delivered by bolus injection, ASO5 distributed broadly throughout the mouse CNS within three weeks (Fig. S2). This finding is consistent with previous reports showing excellent ASO tissue distribution after bolus infusion (and improved pharmacodynamics activity in liver and brain tissues as compared to chronic administration) ${ }^{42,43}$. We found that mice injected with a $30 \mathrm{nmol}$ dose of ASO5 survived and thrived. However, none survived a bolus injection of $50 \mathrm{nmol}$ (Fig. 3a). This low MTD notably limits the dosing scheme and narrows the therapeutic window. To achieve a better balance safety-efficacy, we therefore sought to improve the efficacy and tolerability of ASO5.

ASO5 has a fully modified phosphorothioate (PS) backbone (Fig. 3b,c). Despite its advantages (improved resistance to nucleases and improved cellular uptake), the PS modification also shows increased protein binding that can lead to toxicity ${ }^{44,45}$. Because CSF has relatively low nuclease 
activity ${ }^{46,47}$, we sought to reduce the number of PS linkages to generate a less toxic ASO without compromising activity in the nervous system. ${ }^{48-50}$ Our first attempt consisted of introducing a PS linkage only every other nucleotide. Injection of $50 \mathrm{nmol}$ of this "skipped PS" ASO was tolerated but still was not satisfactory, as mice showed abnormal behavior (slow movement, less reactive to stimuli) in the first 24 hours after treatment. We therefore reduced the PS content within the 5' and 3' MOE-modified "wings" of the ASO, maintaining PS modification throughout the DNA gap. ASO5-1 and ASO5-2 differed only in the presence or absence of a PS linkage between nucleotides 5-6 and 13-14. Both modification patterns showed a higher MTD than ASO5 (Fig. 3a). To explore the mechanism of the active motor phenotypes seen here and to follow up on the improvement observed by mixed backbone patterns, we explored the effect of sugar and phosphate modifications on acute in vivo tolerability ${ }^{51}$. Both mixed-backbone ASOs maintained silencing of the repeatcontaining transcripts in C9-ALS/FTD fibroblasts (Fig. 3d, e), with no significant difference in potency between ASO5, ASO5-1 and ASO5-2.

We administered $30 \mathrm{nmol}$ of each ASO via a bolus ICV injection in C9BAC mice and analyzed efficacy at 8 weeks (Fig. 3f). Each treatment was well tolerated with no adverse side effects or significant weight loss, or changes in blood chemistry (Fig. 3g; Fig. S3b,i). Mice treated with 30nmol of ASO5 and ASO5-2 had a significantly reduced level of V1 and V3 transcripts in cortex and spinal cord as compared to the PBS treated group (Fig. $\mathbf{3 h}, \mathbf{j}$ ) with minimal effect on total transcripts (Fig. 3i,k) showing that absence of PS inter-nucleotide linkages between two MOE modified nucleotides did not impair biological activity in vivo. The silencing was specific and dose dependent (Fig. S3g). By contrast, ASO5-1, which lacked the PS linkage at the junction between a MOE modified and an unmodified DNA nucleotide, achieved only 25\% knock down of V1V3 at a 30nmol dose (Fig. S3c,e). ASO5-2 treatment also reduced polyGP levels in cortex and spinal cord (Fig 31,m). Based on its efficacy and tolerability profile, we selected ASO5-2 as our lead compound.

\section{Sustained and potent effect of mixed-backbone ASO5-2 in the CNS of two C9BAC mouse models.}

We further evaluated ASO5-2 in two ways. First, we obtained a full in vivo dose response of ASO5-2, which revealed an IC50 of $4.75 \mathrm{nmol}$ (Fig. 4a) in the cortex of C9BAC mice three weeks 
after treatment. At the same timepoint, ASO5-2 also significantly reduced levels of polyGP in a dose-dependent manner (Fig. 4b).

We also evaluated the duration of effect of ASO5-2. Our initial studies showed that absence of PS linkages flanked by MOE nucleotides (as in ASO5-2) did not impair biological activity in vitro and in vivo three weeks after treatment. However, since PS linkages also protect ASOs from nuclease degradation, we wondered if the duration of effect of ASO5-2 would be comparable to its fully PS modified parent ASO5. To address this question, we injected C9BAC transgenic mice with 30nmol of ASO5-2 and analyzed the level of V1-V3 transcripts $24 \mathrm{~h}$ or 3, 8 or 20 weeks after injection (Fig. 4c). No effect was observed on the V1-V3 target RNA 24 hours after injection (Fig. 4d). However, a significant, specific dose-dependent reduction of $\sim 80 \%$ of V1V3 transcripts but not total C9 transcripts was observed 3 weeks after injection and, importantly, this was sustained up to 20 weeks in the cortex (Fig. 4d). Analogously, we also observed a sustained reduction in the levels of the polyGP proteins, approaching 90\% reduction even at 20 weeks after the single injection of $30 \mathrm{nmol}$ ASO5-2 (Fig. 4e).

No significant body weight loss or behavioral adverse events (as defined in the methods section) were detected in animals treated with ASO5-2 or the PBS control (Fig. 4f). Likewise, no change in liver, kidney and spleen weight or morphology was observed (Fig. 4g,h). Finally, to further assay the tolerability of ASO5-2 treatment, we analyzed the coordinated motor functions of mice treated with ASO5-2 or injected with vehicle PBS. Seven mice per group were tested in a blinded manner on their rotarod performance weekly after treatment for 19 weeks. No motor deficit was observed in the treated group, underlining the tolerability of ASO treatment (Fig. 4i).

From these observations we concluded that ASO5-2 can be safely administered to transgenic C9BAC mice via intracerebroventricular delivery and that this durably suppresses the offending V1 and V3 transcripts but not the V2 transcript of C9ORF72, as well as the toxic polyGP dipeptides.

\section{ASO5-2 is non-toxic in large animals}

Encouraged by safety and efficacy data in the C9BAC mice, we next obtained additional preclinical toxicity profiles in large animals focusing on ASO5-2 as our lead ASO. As a pilot behavioral study, we performed an intrathecal injection of ASO5-2 at $2 \mathrm{mg} / \mathrm{kg}$ in four sheep. For intrathecal injection in sheep, it was necessary to thread a microcatheter up though the intrathecal 
space and deliver the ASO directly into the cisterna magna (see Methods) ${ }^{51}$. Intracisternal contrast injection and cone beam computed tomography confirmed the correct catheter position prior to ASO injection. At one month, the sheep showed no neurological abnormalities.

We then purchased a batch of GMP-grade ASO5-2 (ChemGenes). The primary sequence of the GMP ASO was confirmed by mass spectrometry (Fig. S4).

We engaged an outside laboratory (Charles River Laboratories) to conduct a GLP safety study of ASO5-2 in cynomolgus monkeys. Twenty-eight normal monkeys averaging $2.5 \mathrm{~kg}$ in weight were treated with $0[n=8], 1.5[n=12]$ or $6.0[n=8]$ mg of ASO5-2. Necropsy was performed at 29 or 90 days (half the animals at each timepoint). Treatment was by intrathecal injection on Days 1, 14, 28 for animals assigned to the Day 29 and Day 90 necropsies and additionally on Days 57 and 85 for animals assigned to the Day 90 necropsy. No behavioral or neurological deficits were observed out to 90 days at either dose; the necropsies did not show pathological findings attributable to ASO5-2 (data not shown).

\section{ASO5-2 suppresses CSF polyGP levels in an individual with the C9ORF72 exon 1 expansion}

Further encouraged by the large animal studies, we administered ASO5-2, which we have designated afinersen, in ascending doses to a single individual with atypical motor neuron dysfunction who harbored a C9orf72 mutation, with appropriate authorization by the Western Institutional Review board and the FDA (IND141673). This individual was a 60-year-old male with a C9ORF72 $\mathrm{G}_{4} \mathrm{C}_{2}$ expansion of approximately 2,400 repeats. He was assessed neurologically because of recurrent left foot-drop induced by distance running followed by slowly progressive weakness in the feet and hands. On examination he demonstrated distal weakness in the legs, diffuse hyporeflexia and sensory loss in the distal legs and feet. Physiological studies documented focally enlarged motor units with evidence of scattered foci of active denervation. CSF analysis revealed elevated levels of polyGP dipeptide repeats (Table 1) but was otherwise benign. As summarized in Fig. 5, on August 29, 2019, he was treated with intrathecal ASO5-2 at 0.5 mg/kg, followed by $1.0 \mathrm{mg} / \mathrm{kg}$ two weeks later. On January 26, 2020, he received a third dose at 1.5 $\mathrm{mg} / \mathrm{kg}$, increased to $2.0 \mathrm{mg} / \mathrm{kg}$ on February $13 ; 2.0 \mathrm{mg} / \mathrm{kg}$ was subsequently administered four more times (Fig. 5). He experienced no medically or neurologically adverse effects from these 
interventions; his laboratory safety studies were unremarkable (Supplementary Table). In multiple CSF evaluations (Table 1), his CSF cell counts, protein and glucose levels were largely unremarkable. At the time of the $4^{\text {th }}$ and $5^{\text {th }}$ CSF analyses, he demonstrated very mild pleocytosis (but not in both CSF tubes on a given day). He also showed a progressive increase in CSF phosphorylated neurofilament heavy and neurofilament light chains (Table 1), which peaked after the $4^{\text {th }} 2.0 \mathrm{mg} / \mathrm{kg}$ dose of ASO5-2 and then partially subsided. The patient's CSF polyGP levels in two samples prior to dosing were in the range $0.01-0.03 \mathrm{ng} / \mathrm{ml}$. After sequential doses of 2.0 $\mathrm{mg} / \mathrm{kg}$, the relative CSF polyGP levels dropped by approximately $80 \%$, correlating with increasing CNS levels of ASO5-2 (Fig. 5, Table 1). During the months of treatment, the patient's ALS functional rating score (ALSFRS-R) was largely stable. Initially it was 38 (48 is normal), decreasing to a nadir of 33 , but then improving to 38 concomitantly with the higher doses of ASO52 .

\section{DISCUSSION 803}

Several years after the identification of the $\mathrm{G}_{4} \mathrm{C}_{2}$ repeat expansion in the gene C9ORF72 as the most common genetic cause of ALS and FTD, multiple investigations have defined potential disease mechanisms. Partial loss of function of C9ORF72 likely contributes to the neurotoxicity of hexanucleotide expansions but does not by itself recapitulate motor neuron degeneration ${ }^{8,9,52}$. In several lines of mice, forced expression of mutant human C9ORF72 in mice also fails to reproduce frank motor neuron pathology but does replicate important molecular signatures, including intranuclear RNA foci and cytoplasmic polydipeptides. These are generated selectively by the two of the three C9ORF72 transcripts, V1 and V3, that harbor hexanucleotide expansions. Together, these observations suggest that an effective therapeutic strategy will be to suppress expression of V1 and V3 to reduce levels of repeat containing RNA and polydipeptides while maintaining baseline levels of V2, which produces the full-length C9orf72 protein and thereby avoids haploinsufficiency. Here, we report that ASOs targeting the intron-repeat 5'junction selectively reduce C9ORF72 repeat containing transcripts V1 and V3 in a dose dependent manner after ICV administration in C9BAC transgenic mice, without reducing the overall level of C9ORF72 V2 transcripts. This intervention significantly reduces numbers of intranuclear RNA foci and depresses level of polyGP dipeptides, which are predicted to be produced from both sense 
and anti-sense strands of the hexanucleotide repeat. Moreover, in a single patient this also reduced polyGP dipeptide levels by approximately $75 \%$.

This report confirms earlier reports from in vitro studies that ASOs can suppress V1 and V3 transcripts in C9-ALS/FTD ${ }^{36}$ and extends our understanding of this therapeutic approach in several important ways. Importantly, we have examined the impact of modifications of the backbone on both the potency and toxicity of the ASOs. Introduction of PS linkages only at the ASO termini and throughout the central DNA region, substantially increased the acute tolerability in the CNS without impairing its potent biological activity. In the course of these studies of ribose and backbone modifications, we compared the toxicities of ICV-bolus vs chronically administered ASOs. It appears that, for comparable doses, delivery by bolus is more effective than by pump. For example, V1-V3 transcripts were reduced $\sim 80 \%$ by ASO5 at $30 \mathrm{nmol}$ delivered by IV bolus, while pump delivery of $50 \mathrm{nmol}$ failed to reduce levels of these transcripts. An analogous difference was observed in ASO therapy in a murine model of spinal muscular atrophy ${ }^{42}$, in which bolus administration proved more effective than chronic infusion.

The administration of ASO5-2 was non-toxic in mice as gauged by behavior and blood panel analysis. A detailed safety study of intrathecally delivered ASO5-2 in cynomolgus monkeys produced no adverse clinical features over 3 months of observation, and no significant pathological or histological findings at 29 and 90 days.

These observations prompted our study of repeated dosing of ASO5-2 via intrathecal delivery in a single patient. This was well tolerated as gauged by clinical examination and the safety profile in blood and CSF (Table 1). With repeated dosing of ASO5-2, we observed a progressive but transient elevation of two CSF biomarkers, phosphorylated neurofilament and neurofilament light chains. We ascribe this to some degree of nerve root irritation provoked by the intrathecal ASO or to some component of the disease not influenced by ASO5-2. This phenomenon is described in the literature in humans but fortunately has not be associated with major neurological consequences ${ }^{50}$. In our case, these biomarkers subsided somewhat in the later CSFs, as the interval between dosing was extended, suggesting a dose-response relation. The patient's clinical metrics improved across the course of therapy, despite the increment in the CSF neurofilament biomarkers. 
Most importantly, our study provides proof-of-concept that ASO therapy in a human can effectively and safely suppress levels of the C9ORF72 transcript that harbor expansions (V1 and V3) without significantly affecting the predominant V2 transcript. That is, this intervention specifically targets not only the mutant allele but the miscreant transcripts generated by that allele. In our models in vitro and in vivo, and in an individual with a C9ORF72 expansion, there was significant reduction in levels of polyGP dipeptide. Suppression of CSF levels of a C9ORF72 polydipeptide has not previously been reported in humans. There is controversy regarding some aspects of the polydipeptide pathology in C9ORF72 ALS. Early studies in drosophila suggested that toxicity arises not from the expanded RNA transcripts but from dipeptides, and particularly those containing arginine ${ }^{19}$. Other reports have also pointed to directly neurotoxic effects of these peptides $23,53-59$, although directly toxic effects of the expanded RNA transcript have also been recently described ${ }^{60}$. Our findings strongly encourage the view that suppressing the expression of the mutant alleles of C9ORF72 may be clinically beneficial, regardless of whether the primary benefit is mediated by reduction in mutant transcripts or polydipeptide levels. Larger clinical trials now underway to assess the clinical impact of therapies that silence C9ORF72 will likely illuminate this hypothesis.

\section{MATERIALS AND METHODS}

\section{Design of Mouse and Human Studies}

The goal of this study was to develop an antisense oligonucleotide (ASO) as a potential clinical therapeutic candidate to treat C9-ALS/FTD. Our experimental approach combined C9 patient derived samples to evaluate selective efficacy in vitro and C9BAC transgenic mice to assess safety, efficacy and duration of effect in vivo. Per experiment, all mice were aged-matched and randomly assigned to control or experimental group. Samples and data were blindly collected, processed and quantified. Molecular and physiological readouts include expression level of V1V3 repeat containing transcripts, all transcripts and peptides, weight of body and organs and motor functions. All outliers were included in data analysis. Exploratory experiments were performed on at least five mice per genotype. Sample size was calculated using the G-power analysis method based on previously defined effect size and standard deviation measuring variability within the sample. 
The project described in this publication was supported by the University of Massachusetts CTSA award number UL1TR001453 from the National Center for Advancing Translational Sciences of the National Institutes of Health. The content is solely the responsibility of the authors and does not necessarily represent the official views of the National Institutes of Health.

The flow chart for the clinical study is presented in Figure 5. This study was conducted with approval from the UMass Medical School and Western Institutional Review Boards (WIRB \# 20183136) and the FDA (IND 141673). Our initial spinal fluid evaluation was performed under the auspices of a Longitudinal Biomarker Study, UMass. Medical School IRB docket \#14341. Patient consent was obtained for these investigations as approved by the IRB.

\section{Antisense Oligonucleotides}

LNA phosphoramidites were synthesized in house using standard methods ${ }^{61}$ from the 3 '-hydroxyl precursors. All other phosphoramidites were purchased from ChemGenes. 0.1M DDTT (ChemGenes) was used as the sulfurizing reagent and $0.25 \mathrm{M}$ BTT (AIC) as the activator. ASOs were synthesized on a Dr. Oligo 48, ABI394, AKTA Oligopilot10 or AKTA Oligopilot 100 synthesizers, according to the required scale. LNA and MOE phosphoramidites were coupled for 8 minutes. Oligonucleotides were deprotected in concentrated aqueous ammonia at $55^{\circ} \mathrm{C}$ for $18 \mathrm{~h}$ and purified using ion-exchange chromatography (eluting with $30 \%$ acetonitrile in water containing increasing gradients of $\mathrm{NaClO}_{4}$ ). Final purification, desalting, concentration and $\mathrm{pH}$ adjustment were effected by diafiltration in an Amicon centrifugal filter. All oligonucleotides were characterized by LCMS.

\section{Dual Luciferase Reporter Assay System}

C9-intron1 containing two $\mathrm{G}_{4} \mathrm{C}_{2}$ motifs (334 nucleotides 5' of the $\mathrm{G}_{4} \mathrm{C}_{2}$ repeat motif and 769 nucleotides in the 3' end) was amplified from blood genomic DNA using the following forward and reverse primers 5'acgtatgcggccgcacgtaacctacggtgtc 3', 5'atacgtgcggcegctaccatcagtcaagtgatg 3 ' and cloned in the psiCHECK ${ }^{\mathrm{TM}}-2$ vector (Promega). C9intron1 expression was measured with the Dual Luciferase Reporter Assay System (Promega) according to the manufacturer instructions.

\section{Cell culture}


HEK293T cells were transfected with $6.5 \mu \mathrm{g}$ of psiCHECK $^{\mathrm{TM}}-2$ vector (Promega) containing C9 intron1 using Lipofectamine 3000 with P3000 reagent according to the manufacturer instructions (ThermoScientific) in T25 flasks. One day after transfection, cells were plated into a 96-well plate in DMEM supplemented with 10\% FBS and treated with the indicated dose of ASO the next day using Lipofectamine RNAiMax Reagent (ThermoScientific). Cells were lysed 48hours after ASO treatment and luciferase signals were quantified.

C9 Patient Derived Fibroblasts. Skin biopsies obtained from two unrelated C9 carriers were cut into small pieces and placed on a culture dish with DMEM supplemented with $15 \%$ fetal bovine serum to allow fibroblasts to expand. ASO treatment was performed on cells plated in $10 \mathrm{~cm}$ dish using Lipofectamine RNAiMax Reagent (ThermoScientific). Total RNA was isolated 72 hours after treatment.

C9BAC Derived Primary cortical neurons. Embryos were removed at E15.5 from pregnant wild-type C57BL/6 females crossed with homozygous C9BAC males. Cortical tissue of each embryo was dissected on ice-cold Hank's Balanced Salt Solution (ThermoScientific). Pooled tissue was minced and digested with $0.05 \%$ Trypsin-EDTA (Life Technology) at $37^{\circ} \mathrm{C}$ for $12 \mathrm{~min}$. Digestion was halted by addition of 10\% FBS/DMEM. Cells were triturated, resuspended in neurobasal media supplemented with Glutamax (ThermoScientific), 2\% penicillin/streptomyocin and B27 supplement (ThermoScientific) and seeded at $0.5 \times 10 \mathrm{E} 6$ cells/well in 6-well plates precoated with poly-ornithine (Sigma). Neurons were treated with ASO at the indicated dose five days after culture and collected 15 days after treatment.

\section{C9ORF72 Bac Transgenic Mice}

C9BAC mice were generated as previously described ${ }^{34}$ and backcrossed to C57BL/6. All experimental protocols and procedures were approved by the University of Massachusetts Medical School Institutional Animal Care and Use Committee.

\section{Stereotaxic Pump Implantation and Bolus Injection of ASO in the Mouse Brain.}

For intracerebroventricular (ICV) infusion of ASO or PBS vehicle through a micro-osmotic pump (Alzet pump model 1007D attached to Alzet brain infusion kit 3), wild-type C57BL/6 or C9BAC transgenic mice were anesthetized and maintained on $2.5 \%$ isoflurane via a nose cone under a stereotaxic frame. Implantation procedure was performed as previously described ${ }^{62}$, with a $3 \mathrm{~mm}$ cannula implantation $0.2 \mathrm{~mm}$ posterior and $1.0 \mathrm{~mm}$ lateral to the right of Bregma. 
For ICV bolus injection mice were anesthetized with isoflurane and placed into a stereotaxic frame. $10 \mu \mathrm{L}$ of sterile PBS or ASO was injected into the right lateral ventricle using the following coordinates: $0.2 \mathrm{~mm}$ posterior and $1.0 \mathrm{~mm}$ lateral to the right from the Bregma and lowered to a depth of $3 \mathrm{~mm}$.

\section{Mouse Behavior Monitoring}

Over the course of treatment and immediately prior to sacrifice, each animal was blindly weighed and evaluated weekly by a trained observer for adverse events, defined as any behavior not typical in a naïve matched control animal, including, but not limited to: limb clasping, tremors, abnormal respiration, paralysis, spasticity, impaired reflex, hyperactivity and lethargy.

\section{Rotarod}

Coordinated motor functions were assessed in control and treated mice using the rotarod test as previously described ${ }^{34}$. Briefly, mice were tested weekly beginning two weeks prior to $\mathrm{ASO} /$ vehicle administration and ending at the week of sacrifice. Each animal was given three trials on a 4-40rpm accelerating rotarod for 5 minutes with a one minute inter trial interval. Latencies to fall for each animal was automatically recorded by a computer and plotted as a mean +/- SEM.

\section{Blood Biochemistry}

Whole blood samples were collected after cardiac puncture (terminal procedure). Blood biochemistry was performed using the VetScan Comprehensive diagnostic profile (Abaxis, Union City, CA).

\section{Southern Blot}

Southern blot was performed on $10 \mu \mathrm{g}$ genomic DNA isolated using Gentra Puregene Tissue kit (Qiagen). DNA was digested overnight with AluI and DdeI at $37^{\circ} \mathrm{C}$ and separated by electrophoresis on a $0.6 \%$ agarose gel, transferred to a positively charged nylon membrane (Roche Applied Science), cross-linked by UV, and hybridized overnight at $55^{\circ} \mathrm{C}$ with a digoxigeninlabeled $\mathrm{G}_{2} \mathrm{C}_{4}$ DNA probe in hybridization mix buffer (EasyHyb, Roche). The digoxigenin-labeled probe was detected with anti-digoxigenin antibody and CDP-Star reagent as recommended by the manufacturer (Roche).

\section{RNA Extraction and Quantitative RealTime-PCR}


Total RNA was isolated from snap frozen cortex or spinal cord tissue using Trizol (ThermoScientific) and subsequently treated with DNase I (Qiagen). One $\mu \mathrm{g}$ of total RNA was reverse transcribed into cDNA using random hexamers and MultiScribe reverse transcriptase (ThermoScientific) following the manufacturer's instructions. Quantitative PCR was performed on a StepOnePlus Real-Time PCR system using SYBR Green Master Mix (Applied Biosystems) and $0.2 \mu \mathrm{M}$ of forward and reverse primers as described in ${ }^{54} \mathrm{Ct}$ values for each sample and gene were normalized to $G A P D H$. The $2 \exp (-\triangle \Delta \mathrm{Ct})$ method was used to determine the relative expression of each target gene.

\section{Fluorescence in situ Hybridization (FISH)}

FISH was performed as previously described ${ }^{54}$ using a 5 ' end Cy3-conjugated $\left(\mathrm{G}_{2} \mathrm{C}_{4}\right)_{4}$ oligonucleotide DNA probe at $55^{\circ} \mathrm{C}$ in hybridization buffer containing formamide 40\%/2XSSC/0.1\% Tween-20/DNA salmon sperm. Samples were then washed twice in prewarmed wash buffer (formamide $40 \% / 2 \mathrm{XSSC} / 0.1 \%$ Tween-20) and in stringency wash buffer $(0.2 \mathrm{XSSC} / 0.1 \%$ Tween 20$)$ at $55^{\circ} \mathrm{C}$. Samples were then mounted in Prolong Gold Antifade reagent with DAPI (ThermoScientific). Confocal images were taken with a Leica TCS SP5 II laser scanning confocal microscope and processed with Leica LAS AF software.

\section{Detection of poly(GP) and poly(PR)}

Rabbit polyclonal anti-poly GP and PR antibodies were generated to the repeat motif $\left(\mathrm{GP}_{8}, \mathrm{PR}_{8}\right)$ by New England Peptide. Poly(GP) or poly(PR) levels in lysates were measured using a sandwich immunoassay that utilizes Meso Scale Discovery (MSD) electrochemiluminescence detection technology. Tissue samples were lysed in RIPA buffer supplemented with $1 \times$ Roche complete protease inhibitor and 1X Halt Phosphatase Inhibitor Cocktail (ThermoScientific) using TissueLyserII (Qiagen) followed by sonication on ice. Samples were gently homogenized on a rocker at $4^{\circ} \mathrm{C}$ for $30 \mathrm{~min}$. Debris were removed by centrifugation $\left(15 \mathrm{~min}, 14000 \mathrm{~g}, 4^{\circ} \mathrm{C}\right)$ and the supernatant collected. Total protein concentration was determined using the BCA Protein Assay Kit (Thermo Scientific). $50 \mu \mathrm{g}$ of total protein diluted in PBS-Tween supplemented with 10\% fetal bovine serum was loaded per well in duplicate wells. Serial dilutions of recombinant (GP) 8 or $(\mathrm{PR})_{8}$ spiked in wild-type $\mathrm{C} 57 \mathrm{Bl} / 6$ brain protein extracts were used to prepare the standard curve. Response values corresponding to the intensity of emitted light upon electrochemical stimulation of the assay plate using the MSD QUICKPLEX SQ120 were acquired and background corrected using the average response from lysates obtained from wild-type C57Bl/6 brain extract. 


\section{Detection of pNFH and NFL}

Neurofilament light chain (NFL) and phosphorylated neurofilament heavy chain (pNFH) were detected in CSF samples using sandwich immunoassays and the MSD QUICKPLEX SQ120. For pNFH measures, the Iron Horse Diagnostics clinically validated assay was used with a monoclonal anti-pNFH antibody as capture and sulfo-tagged polyclonal anti-pNFH antibody for detection. Purified pNFH was used to generate a calibration curve. All assays were performed in triplicate with a coefficient of variation $(\mathrm{CV})<5 \%$ for all samples. The lower limits of quantification of the pNFH assay is $1.2 \mathrm{pg} / \mathrm{ml}$ in CSF. For NFL measures, a R-PLEX human NFL MSD assay kit was used (MSD catalog \# F217X) following manufacturer specifications. All assays were performed in triplicate with a $\mathrm{CV}<5 \%$ for all samples. The lower limits of quantification of the NFL assay is $2.0 \mathrm{pg} / \mathrm{ml}$.

\section{Immunohistochemistry}

Brains were rapidly removed from euthanized animals. The contralateral hemibrain was post-fixed in $10 \%$ formalin. Paraffin-embedded or cryoprotected blocks were cut in $10 \mu \mathrm{m}$ thick sagittal sections. Slides were permeabilized with Triton $0.1 \%$ for $10 \mathrm{~min}$. Non-specific antibody binding was blocked by incubation with $10 \%$ goat serum in PBS/Tween $0.01 \%$ for one hour. Primary antibodies were diluted in blocking solution and sections were incubated overnight at $4^{\circ} \mathrm{C}$. After three washes in $\mathrm{PBS} /$ Tween $0.01 \%$, sections were incubated with Alexa fluor-488 or -546 conjugated secondary antibodies diluted in PBS for one hour at room temperature. Autofluorescence was quenched by slide immersion in $0.5 \%$ Sudan BlackB in $70 \%$ ethanol and cell nuclei were stained with DAPI. Primary antibodies used: mouse anti-NeuN (1:500, Millipore), rabbit anti-P/S ASO (1:500, in house). Briefly, a rabbit polyclonal antibody raised in house by inoculating two female New Zealand White rabbits with a fully-PS-modified, KLH-conjugated ASO. Boosts and bleeds were carried out at regular intervals over one year, and antisera were used for histology (Moazami and Watts, in preparation).

\section{Haematoxylin and eosin (HE) staining}

8 to $10 \mu \mathrm{m}$ thick sections of mouse liver and kidney were cut from formalin-fixed, paraffin embedded blocks. Standard HE staining was performed.

\section{Mass spectrometric characterization of C9orf72 antisense oligonucleotide}


The sequence and modification pattern of the clinical C9orf72 antisense oligonucleotide (ASO) was confirmed by mass spectrometric (MS) analysis performed by nanoflow electrospray ionization (i.e., nanospray) on a Fourier transform ion cyclotron resonance (FTICR) mass spectrometer (vide infra for conditions). The multiply charged ions observed in negative ion mode (Figure S4a) provided a monoisotopic molecular mass of $6461.1894 \mathrm{u}$, which matched very closely the expected value of 6461.1888 Da calculated from sequence. In subsequent experiments, the [M $-4 \mathrm{H}]^{4-}$ molecular ion at $1615 \mathrm{~m} / \mathrm{z}$ was submitted to tandem mass spectrometry $\left(\mathrm{MS}^{2}\right)$ to achieve sequence confirmation (vide infra). Upon isolation in the mass selective quadrupole (Q) and activation by collisions with Ar in the collision cell (q), the precursor ion exhibited typical fragment series corresponding to the central region of the construct, which confirmed the sequence spanning from A7 to C13 (Figure S4b). Distinctive signals were additionally detected at 1179 and $1971 \mathrm{~m} / \mathrm{z}$, which were assigned to fragments corresponding to the entire G1:T6 and G14:C18 moieties, respectively. In turn, each of these first-generation fragments were individually isolated and activated in the FTICR cell to complete MS $^{3}$ determinations (Figure S4c-d). These experiments provided abundant second-generation fragments that confirmed the sequences of the construct's terminal regions.

All analyses were carried out on a Bruker (Billerica, MA) 12T solariX FTICR equipped with a 12-tesla superconducting magnet and a home-built ion source that enabled static nanospray operations. No sample desalting or chromatographic steps were employed. All samples consisted of a $4 \mu \mathrm{M}$ solution of C9orf72 antisense oligonucleotide in $150 \mathrm{mM}$ ammonium acetate ( $\mathrm{pH}$ 7.4) and $10 \%$ volume of 2-propanol. In each analysis, a $5 \mathrm{~mL}$ aliquot was loaded onto a quartz emitter prepared in-house. A stainless steel wire was inserted from the back end to provide the voltage necessary to achieve a stable spray. The instrument was calibrated by using a $1 \mathrm{mg} / \mathrm{mL}$ CsI solution that provided an accuracy of $87 \mathrm{ppb}$. Detection was accomplished in broadband mode, which afforded a typical 230,000 resolution. The first activation step was carried out in the collision cell (q) of the instrument, which was flooded with a low pressure of Ar and subjected to an $18.5 \mathrm{~V}$ activation voltage. The second activation step was carried out in the FTICR cell by irradiating selected ions with a frequency that was $250 \mathrm{~Hz}$ off-resonance and $0.65-1.29 \%$ power. The data were processed by using the DataAnalysis package provided by 
Bruker. The results were interpreted by using the Mongo Oligo Mass Calculator v2.08 available at https://mods.rna.albany.edu/masspec/Mongo-Oligo.

\section{Statistical Analysis}

All data were graphed as mean \pm SEM and analyzed using GraphPad Prism Software (Version7). Tests between two groups used the two-tailed student-t test. Tests between multiple groups used one-way analysis of variance (ANOVA) corrected with Bonferroni multiple comparison post-hoc test. $* \mathrm{p}<0.05, * * \mathrm{p}<0.01, * * * \mathrm{p}<0.001, * * * * \mathrm{p}<0.0001$, ns not significant

Acknowledgments: The authors thank the Brown and Watts labs, Wave Life Sciences, the Animal Medicine and DERC Morphology Cores and F. Ladam for advice, technical support and manuscript review. Funding: This work was funded by the NIH (R01 NS111990 to RHB and JKW), the Angel Fund for ALS Research, and the Ono Pharmaceutical Foundation (Breakthrough Science Award to JKW). RHB also acknowledges funding from ALSOne, ALS Finding a Cure, the Cellucci Fund for ALS Research and the Max Rosenfeld Fund. Author contributions: RB conceived project; HT, RHB, JKW, MPM designed experimental plan; MPM performed oligo synthesis; MPM, CP, JM and AW supported mouse experiments; HT, HY and NW processed cell and mouse tissue experiments; MPM raised the PS-targeted antibody and performed brain staining; TK and DF verified the sequence of the clinical ASO; NS and CD prepared the drug product for clinical use; RHB, DM-Y and CD supported clinical work including preparing consent forms; HGE, MPM, MM and RMK supported sheep studies; MS and NW evaluated ASO levels in patient CSF and monkey tissues; HT, RHB and JKW wrote manuscript; RHB and JKW supervised project. We also acknowledge the NEALS Biorepository for providing all or part of the biofluids from the ALS, healthy controls and non-ALS neurological controls used in this study. The project described in this publication was supported in part by the University of Massachusetts CTSA award number UL1TR001453 from the National Center for Advancing Translational Sciences of the National Institutes of Health. The content is solely the responsibility of the authors and does not necessarily represent the official views of the National Institutes of Health. Competing interests: The authors have filed a patent related to this research. Data and materials availability: C9BAC mice and PS-targeted polyclonal antibody are available by contacting the investigators 
Table 1 Cerebrospinal Fluid Parameters in Relation to Dosing of ASO5-1 in Patient

\begin{tabular}{|c|c|c|c|c|c|c|c|c|c|c|c|c|c|c|c|c|c|c|c|c|c|}
\hline \multirow[b]{2}{*}{ Date } & \multirow[b]{2}{*}{ Wks } & \multicolumn{2}{|c|}{ Chems $^{1}$} & \multirow{2}{*}{$\begin{array}{l}\text { ASO }^{2} \\
\text { Dose }\end{array}$} & \multirow{2}{*}{$\begin{array}{c}\text { ASO } \\
\text { Level }^{3}\end{array}$} & \multirow{2}{*}{$\begin{array}{l}\text { GP } \\
\text { Rel }\end{array}$} & \multirow[b]{2}{*}{ pNFH $^{5}$} & \multirow[b]{2}{*}{$\mathrm{NFL}^{6}$} & \multicolumn{2}{|c|}{ Tube 1} & \multicolumn{3}{|c|}{ Tube $\mathbf{n}$} & \multicolumn{4}{|c|}{ Differential \% (Tube 1) } & \multicolumn{4}{|c|}{ Differential \% (Tube n) } \\
\hline & & Glu & Pro & & & & & & WBC ${ }^{7}$ & $\mathrm{RBC}^{8}$ & WBC & RBC & n & \# cells & Lymphs & Monos & Polys & \# cells & Lymphs & Monos & Polys \\
\hline 19-May-16 & & 71 & 56 & & & 1.00 & & & 2 & 37 & 1 & 6 & 3 & 16 & 31 & 63 & 6 & 100 & 45 & 54 & 1 \\
\hline 26-Aug-19 & 0.0 & & 50 & 0.5 & $0]$ & 1.00 & 253.8 & $2,249.8$ & & & 0 & 0 & 4 & 5 & 100 & & & & & & \\
\hline 9-Sep-19 & 2.0 & 65 & 48 & 1.0 & 11.74 & 1.05 & 309.2 & $2,478.2$ & & & 1 & 2 & 4 & 50 & 36 & 64 & & & & & \\
\hline 7-Oct-19 & 6.0 & 62 & 52 & & 19.88 & 1.08 & 444.8 & $2,840.3$ & & & 1 & 0 & 4 & 18 & 67 & 33 & & & & & \\
\hline $16-\operatorname{Jan}-20$ & 20.4 & 69 & 54 & 1.5 & 1.68 & 0.62 & 353.4 & $2,922.0$ & 1 & 3 & 2 & 0 & 4 & 9 & 67 & 33 & & 4 & 50 & 50 & \\
\hline $30-\operatorname{Jan}-20$ & 22.4 & 71 & 54 & & 20.49 & 0.62 & 299.4 & $3,212.4$ & 3 & 211 & 1 & 2 & 4 & 100 & 58 & 36 & 6 & 36 & 67 & 33 & \\
\hline $13-F e b-20$ & 24.4 & 59 & 55 & 2.0 & 15.05 & 0.53 & 350.2 & $3,078.4$ & 1 & 3 & 1 & 1 & 3 & 27 & 74 & 26 & & 11 & 55 & 45 & \\
\hline $26-\mathrm{Feb}-20$ & 26.3 & 65 & 65 & & 42.52 & 0.37 & 516.7 & $3,687.9$ & 1 & 1 & 1 & 1 & 3 & 42 & 45 & 55 & & 50 & 44 & 56 & \\
\hline 9-Mar-20 & 28.0 & 64 & 52 & 2.0 & 26.08 & 0.38 & 442.4 & $4,137.3$ & 3 & 1 & 1 & 1 & 3 & 66 & 58 & 42 & & 81 & 62 & 38 & \\
\hline 23-Apr-20 & 34.4 & 67 & 62 & & 17.74 & 0.24 & 810.8 & $11,269.3$ & 4 & 173 & 8 & 1 & 3 & 100 & 41 & 58 & 1 & 100 & 54 & 46 & \\
\hline 4-May-20 & 36.0 & 65 & 66 & 2.0 & 14.4 & 0.21 & $1,156.3$ & $13,626.6$ & 11 & 0 & 3 & 2 & 3 & 100 & 58 & 42 & 6 & 6 & 67 & 33 & \\
\hline 27-Jul-20 & 48.0 & 67 & 49 & 2.0 & 9.33 & 0.23 & $1,337.9$ & $2,790.5$ & 8 & 4 & 3 & 7 & 2 & 23 & 78 & 22 & & 100 & 65 & 33 & 2 \\
\hline $20-0 \mathrm{ct}-20$ & 60.1 & 66 & 40 & 2.0 & 8.86 & ND & 966.2 & $10,361.5$ & 2 & 1 & 1 & 0 & 3 & 19 & 47 & 53 & & 58 & 62 & 48 & 3 \\
\hline & 1 & $\mathrm{mg} / \mathrm{dl}$ & & & & 4 & $\mathrm{ng} / \mathrm{ml}$ & As compo & ared to $b$ & baseline & $\mathrm{e}: \sim 0.0$ & $1-0.0$ & 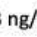 & & & & cells $/ n$ & $\mathrm{~nm}^{3}$ & & & \\
\hline & 2 & $\mathrm{mg} / \mathrm{dl}$ & & & & 5 & $\mathrm{pg} / \mathrm{ml}$ & Normal 2- & $300 ; A L S$ & $S 250-1$ & 12,000 & & & & & & cells $/ \mathrm{n}$ & $\mathrm{nm}^{3}$ & & & \\
\hline & 3 & $\mathrm{ng} / \mathrm{ml}$ & & & & 6 & $\mathrm{pg} / \mathrm{ml}$ & Normal 10 & $00-1,500$ & ; ALS 1, &, 000 - & 20,00 & & & & & not de & tected & & & \\
\hline
\end{tabular}



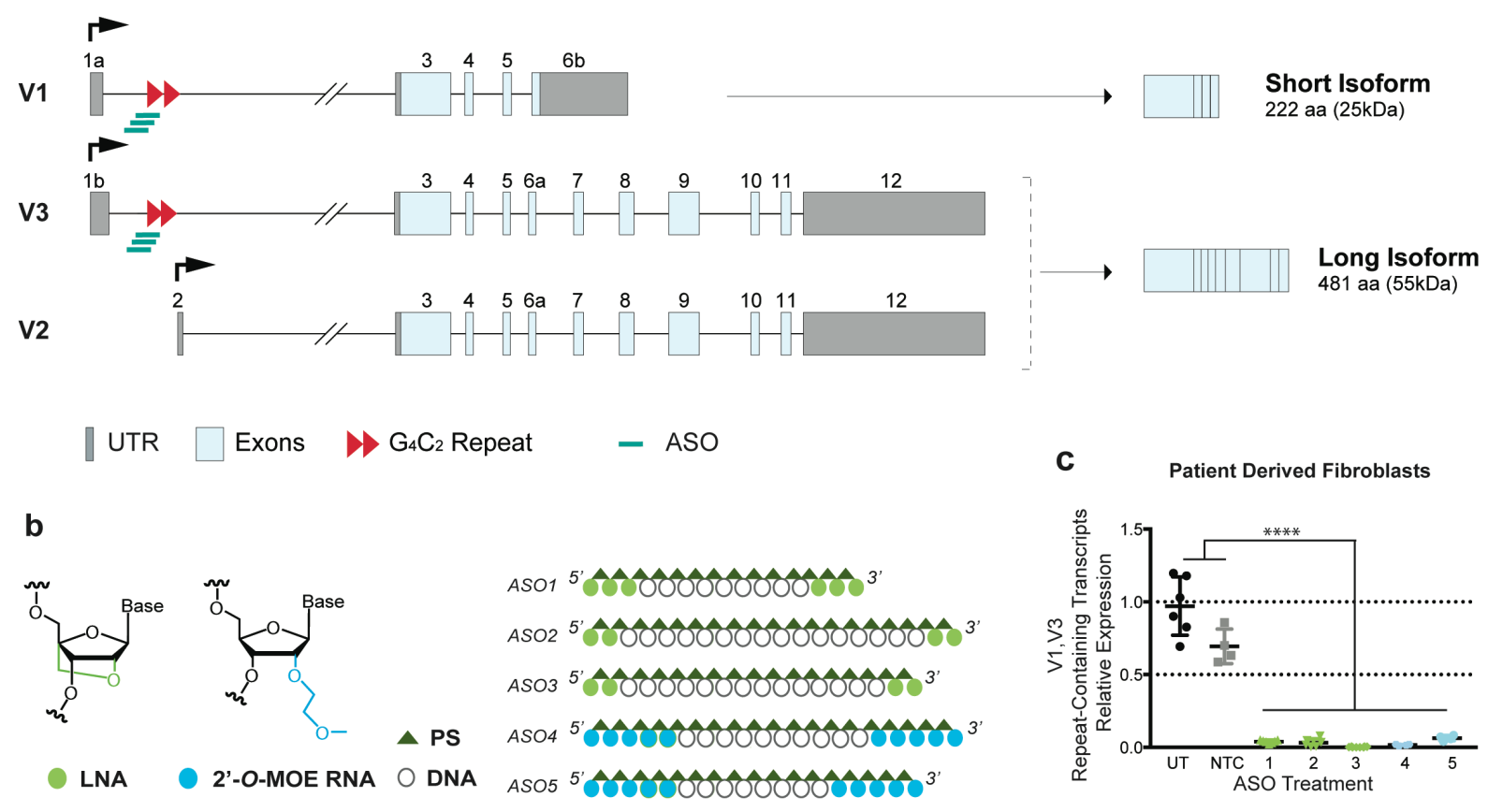

C Patient Derived Fibroblasts

d

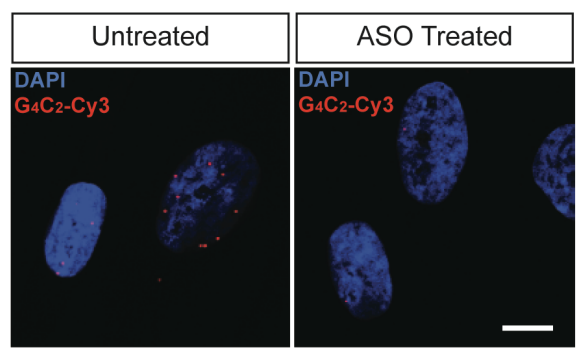

e

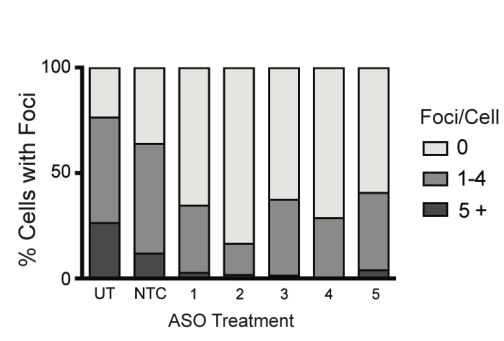

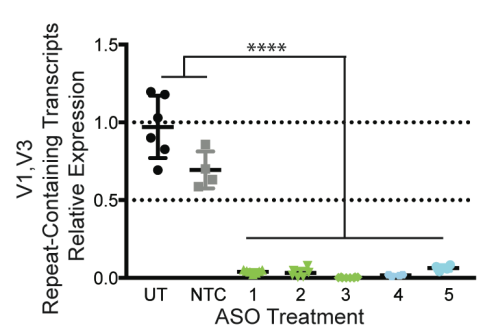

f

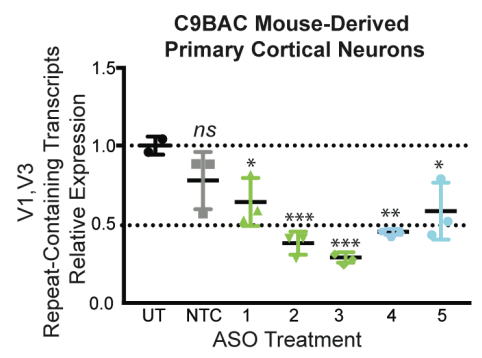

Figure 1. $\mathrm{G}_{4} \mathrm{C}_{2}$ targeting $\mathrm{LNA}$ and MOE modified ASO reduces the C9ORF72 repeat containing transcripts in patient derived fibroblasts and C9BAC mouse derived neurons.

(a) Schematic of C9ORF72 transcript variants and of the two protein isoforms as named in PubMed. The repeat expansion (red triangles) located in the first intron is expressed in variants 1 and 3 (V1-V3). Variant 2 (V2), the most abundant, starts form a different transcription start site (black arrow) and does not include the repeat expansion. V2 and V3 encode the main C9ORF72 protein isoform. Grey boxes represent untranslated regions (UTR); light blue boxes, exons; black lines, introns. ASOs used in this study (green bars) target the intronic region flanking the repeat expansion. (b) Schematic of ASOs and their chemical modifications used in this study: green circle, locked nucleic acid (LNA); blue circle, 2'-O-2-methoxyethyl RNA (2'-O-MOE); empty circle, DNA; dark green triangle, phosphorothioate nucleotide linkage (PS). (c) LNA and 2'-OMOE ASOs significantly reduce the level of repeat containing transcripts at a dose of 100nM 72 hrs after lipid-mediated delivery in patient derived fibroblasts as measured by qRT-PCR. (d) 
Representative images of RNA foci visualized by FISH in patient derived fibroblasts untreated or treated with ASO for $72 \mathrm{hrs}$. (e) \% of nuclei without foci (light grey), with 1-4 foci (dark grey) or with more than 5 foci (black) when cells were treated with no ASO, a non-targeting control ASO (NTC), LNA modified ASOs (ASOs 1-3) or 2'-O-MOE modified ASOs (ASOs 4-5); 50 cells examined for each group. (f) LNA and 2'-O-MOE ASOs significantly reduce the level of repeatcontaining transcripts after two weeks treatment with $1 \mu \mathrm{M}$ ASO without lipid assistance (gymnotic delivery) in primary cortical neurons derived from C9BAC mice. 


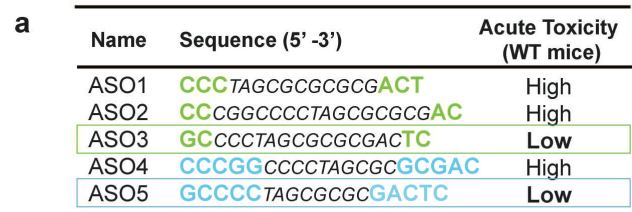

b

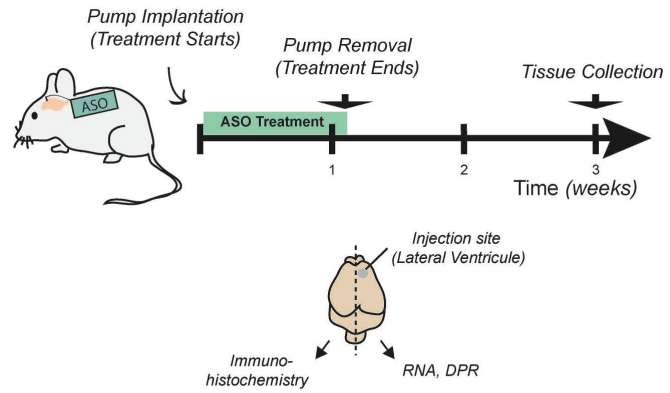

C
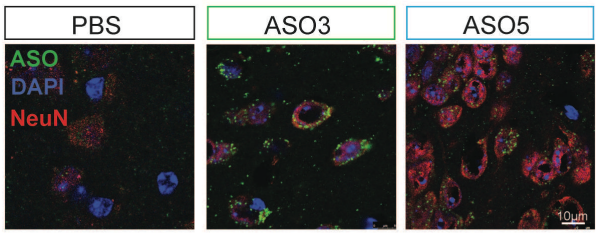
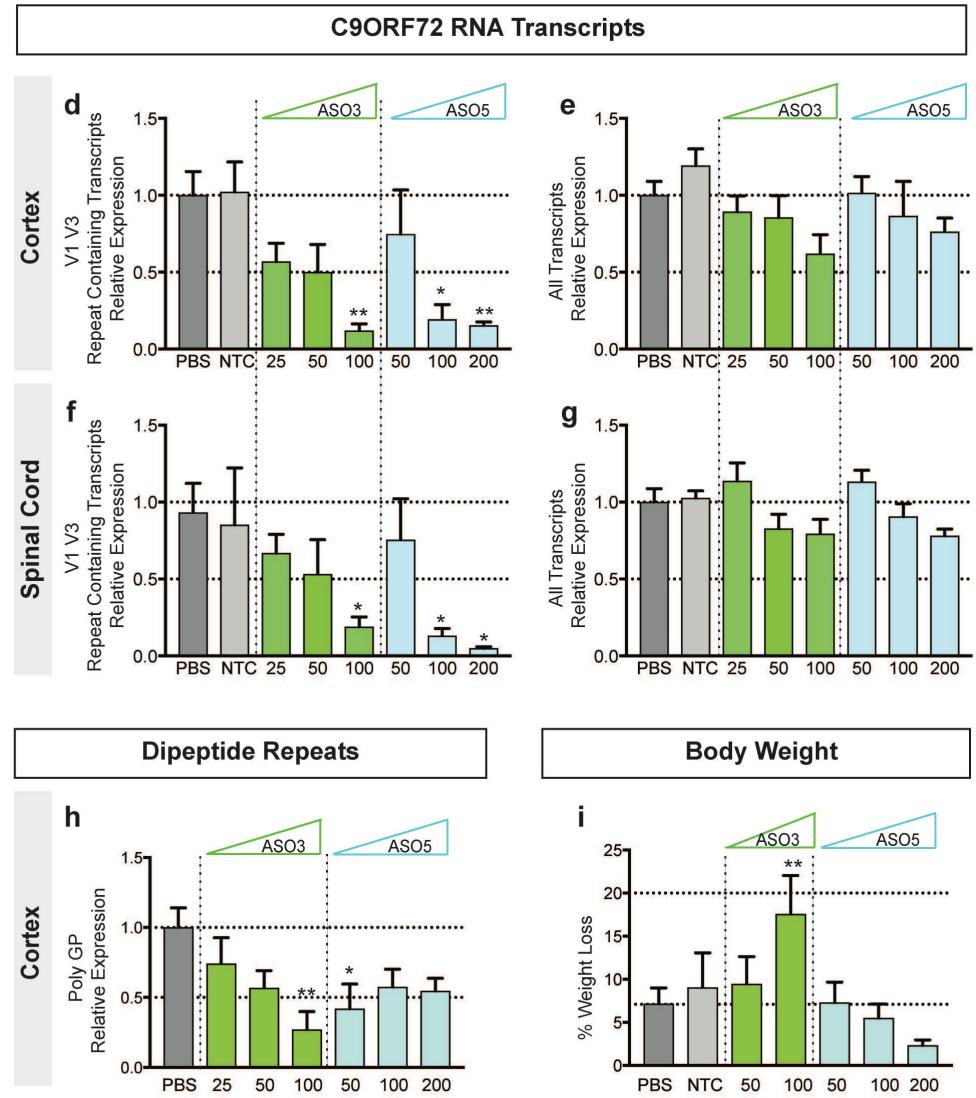

Figure 2. $\mathrm{G}_{4} \mathrm{C}_{2}$ targeting LNA and MOE modified ASOs selectively reduces the C9ORF72 repeat containing transcripts and peptides after CNS infusion in C9BAC mice

(a) ASO sequences and their in vivo response in 4-6-month-old wild-type mice after ICV bolus injection: bold green, LNA; bold blue, MOE; italic black, DNA. (b) Schematic of experimental design in heterozygous C9BAC mice. Vehicle control (PBS), a non-targeting control ASO (NTC) and 25 to 200nmol of ASO3 and ASO5 were infused into the right lateral ventricle of 5-6-monthold C9BAC mice over 10 days. Brain and spinal cord were harvested and dissected two weeks after pump removal for RNA and DPR analysis (spinal cord and ipsilateral brain hemisphere), and ASO staining (contralateral brain hemisphere). (c) ASO3 and ASO5 (green) are taken up in neurons (red) - nuclei counterstaining in blue. (d-g) Expression of V1-V3 repeat containing transcripts $(\mathbf{d}, \mathbf{f})$ and all transcripts $(\mathbf{e}, \mathbf{g})$ in cortex and spinal cord quantified by qRT-PCR in mice infused with PBS (dark grey), NTC ASO (light grey), ASO3 (green) or ASO5 (blue) at the indicated dose. For each dose level, $n=5-7$, except NTC group $(n=3)$. (h) Relative expression of polyGP in the cortex of mice treated with ASO3 (green) and ASO5 (blue) assayed by sandwich immunoassay. Data are represented as mean \pm SEM. (i) $\%$ of body weight loss at end point relative to before treatment. 

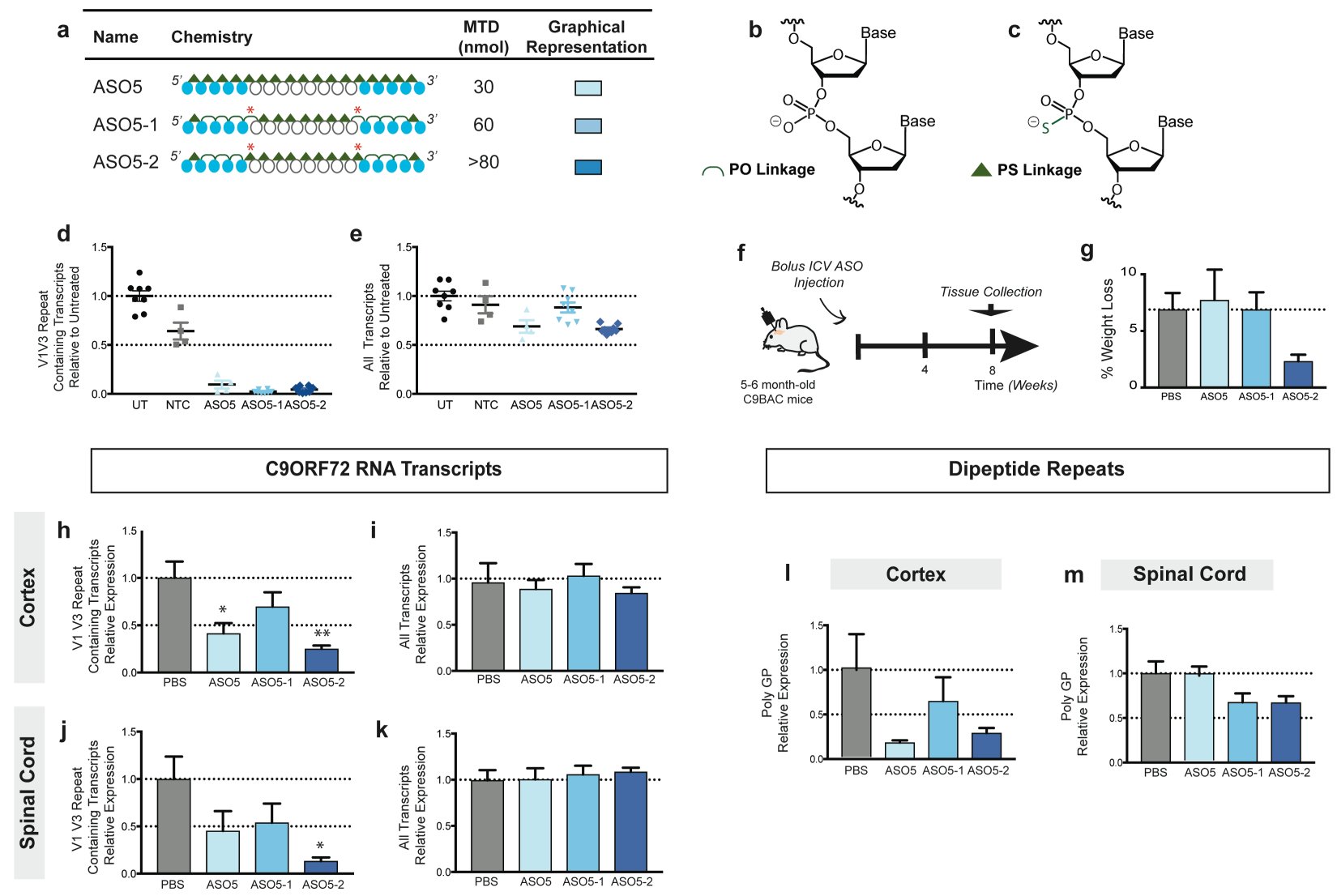

Figure 3. Reducing the phosphorothioate content of ASO5 improves its safety profile without reducing its biological activity after CNS administration in C9BAC mice

(a) Chemistry and maximum tolerated dose (MTD) in nmol of ASO5 and its mixed-backbone derivatives (ASO5-1 and ASO5-2). Maximum Tolerated Doses (MTD) were evaluated in vivo in 5-6-month-old wild-type C57Bl/6 mice for up to 8 weeks after ICV bolus injections. (a-b) green hemi-circle, phosphodiester linkage (PO); (a-c) green triangle, phosphorothioate linkage (PS). In (a) closed blue circle, 2'-O-MOE; open circle, DNA: red star highlights the position of PS/PO difference between ASO5-1 and ASO5-2. (d-e) Effect of 100nM of a mixed PS/PO on ASO5 efficacy in patient-derived fibroblasts on expression of V1, V3 repeat-containing transcripts (d) or all transcripts (e). (f) Schematic of experimental design in heterozygous C9BAC mice. Vehicle control (PBS), ASO5, ASO5-1 or ASO5-2 were injected into the right lateral ventricle of 5-6 month old C9BAC mice. Brain and spinal cord were harvested and dissected eight weeks after treatment for RNA and DPR analysis. (g) No significant change in body weight was observed eight weeks after treatment when mice were treated with ASO. (h-m) Expression of V1-V3 repeat containing transcripts $(\mathbf{h}, \mathbf{j})$ and all transcripts $(\mathbf{i}, \mathbf{k})$ in cortex and spinal cord quantified by qRTPCR and polyGP $(\mathbf{l}, \mathbf{m})$ in mice treated with PBS (dark grey), ASO5 (light blue), ASO5-1 (medium blue) and ASO5-2 (dark blue) eight weeks after administration of 30nmol of each ASO. For each ASO group, $\mathrm{n}=5-7$. 

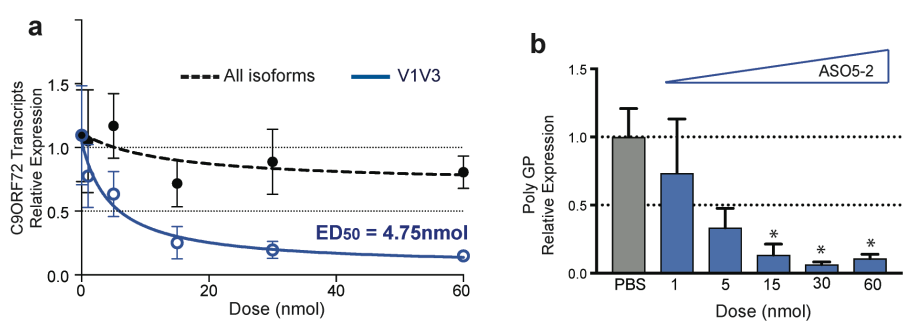

$\mathbf{C}$

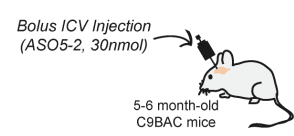

Harvest after:
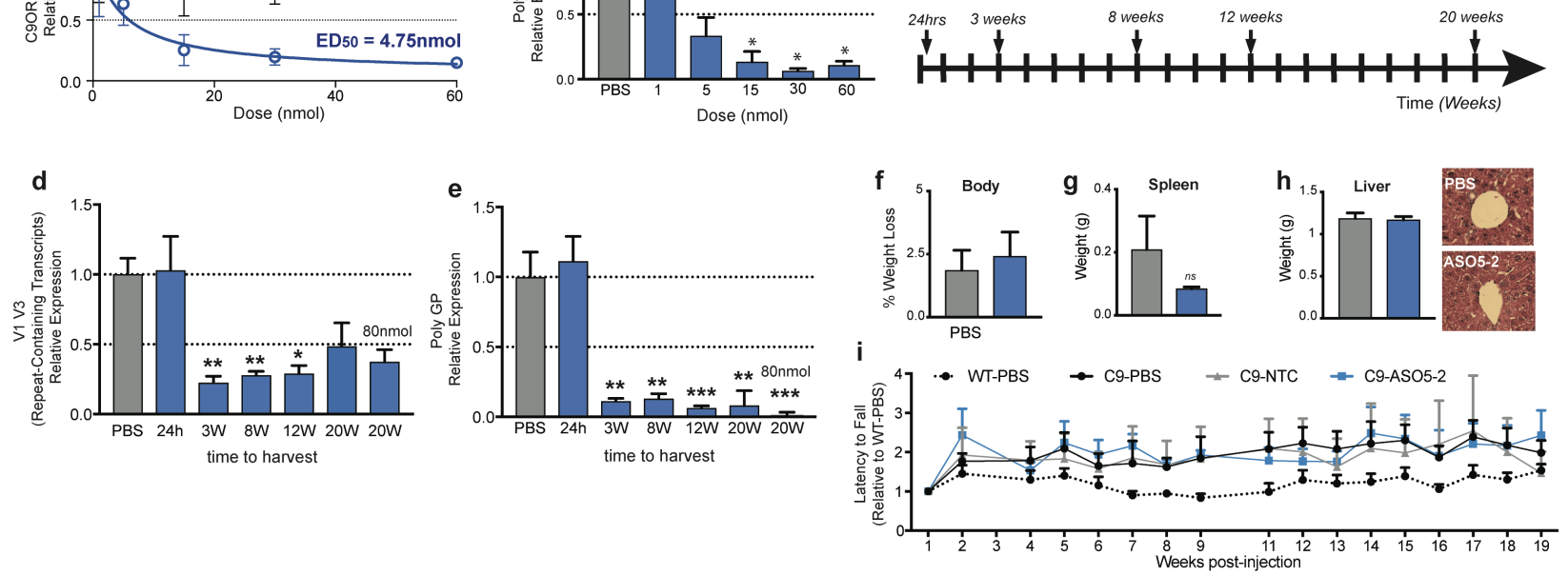

Figure 4 Sustained and potent effect of ASO5-2 in the CNS of C9BAC mice.

(a-b) Dose-dependence of gene silencing by ASO5-2 in heterozygous C9BAC mice. Vehicle control (PBS) and a single injection of 1, 5, 15, 30, or $60 \mathrm{nmol}$ of ASO5-2 was administered into the lateral ventricle of 5-6-month-old heterozygous C9BAC mice. Expression of V1-V3 repeatcontaining transcripts and all transcripts (a) and polyGP (b) was analyzed 3 weeks after injection. (c-i) A time course experiment was performed in heterozygous C9BAC mice treated with 30nmol of ASO5-2, tissues were collected and analyzed at $24 \mathrm{~h}$ or 3, 8, 12 and 20 weeks after treatment (schematic shown in panel c); an $80 \mathrm{nmol}$ dose was also harvested at 20 weeks only. Expression of V1,V3 repeats containing transcripts (d) and polyGP (e) was analyzed in cortex $24 \mathrm{~h}$ or 3, 8, 12 or 20 weeks after a single dose injection of ASO5-2. No significant change in body weight (f), spleen (g) liver (h) and kidney (not shown) weight or morphology was observed after treatment with ASO5-2 (blue) relative to PBS (grey) 8 weeks after treatment. ASO treatment did not alter motor performance on a 5-minute accelerating rotarod task during the treatment course (i). For each treatment or dose group, $n=6-7$. 


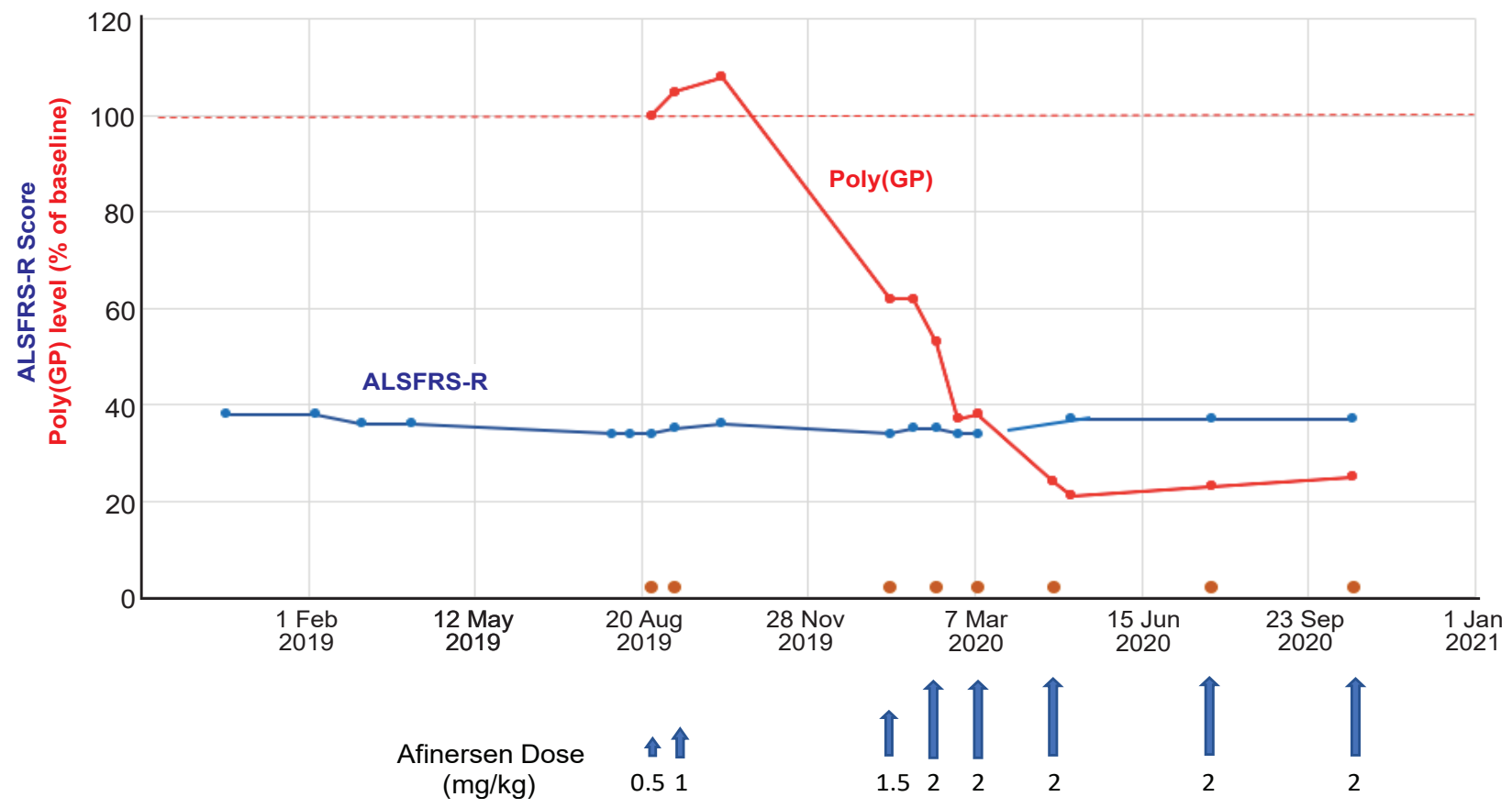

Figure 5. Clinical summary and afinersen (ASO5-2) dosing. A single patient received multiple doses of ASO5-2 as shown below the graph. The patient's ALSFRS-R score before and during treatment is shown as a blue line and points. The patient's poly(GP) DPR level, relative to the baseline (100\%) is shown as a red line and points. 


\section{References}

1. Dejesus-Hernandez, M., et al. Expanded GGGGCC Hexanucleotide Repeat in Noncoding Region of C9ORF72 Causes Chromosome 9p-Linked FTD and ALS. Neuron 72, 245-256 (2011).

2. $\quad$ Renton, A.E., et al. A Hexanucleotide Repeat Expansion in C9ORF72 Is the Cause of Chromosome 9p21Linked ALS-FTD. Neuron 72, 257-268 (2011).

3. Gijselinck, I., et al. A C9orf72 promoter repeat expansion in a Flanders-Belgian cohort with disorders of the frontotemporal lobar degeneration-amyotrophic lateral sclerosis spectrum: a gene identification study. Lancet Neurol 11, 54-65 (2012).

4. Cook, C. \& Petrucelli, L. Genetic Convergence Brings Clarity to the Enigmatic Red Line in ALS. Neuron 101, 1057-1069 (2019).

5. Swinnen, B., Robberecht, W. \& Van Den Bosch, L. RNA toxicity in non-coding repeat expansion disorders. EMBO J 39, e101112 (2020).

6. Rodriguez, C.M. \& Todd, P.K. New pathologic mechanisms in nucleotide repeat expansion disorders. Neurobiol Dis 130, 104515 (2019).

7. Amick, J. \& Ferguson, S.M. C9orf72: At the intersection of lysosome cell biology and neurodegenerative disease. Traffic 18, 267-276 (2017).

8. Belzil, V.V., et al. Reduced C9orf72 gene expression in c9FTD/ALS is caused by histone trimethylation, an epigenetic event detectable in blood. Acta Neuropathol 126, 895-905 (2013).

9. Waite, A.J., et al. Reduced C9orf72 protein levels in frontal cortex of amyotrophic lateral sclerosis and frontotemporal degeneration brain with the C9ORF72 hexanucleotide repeat expansion. Neurobiol Aging 35, 1779 e1775-1779 e1713 (2014).

10. Zhu, Q., et al. Reduced C9ORF72 function exacerbates gain of toxicity from ALS/FTD-causing repeat expansion in C9orf72. Nat Neurosci 23, 615-624 (2020).

11. O'Rourke, J.G., et al. C9orf72 is required for proper macrophage and microglial function in mice. Science 351, 1324-1329 (2016).

12. Burberry, A., et al. Loss-of-function mutations in the C9ORF72 mouse ortholog cause fatal autoimmune disease. Sci Transl Med 8, 347ra393 (2016).

13. Shi, Y., et al. Haploinsufficiency leads to neurodegeneration in C9ORF72 ALS/FTD human induced motor neurons. Nat Med 24, 313-325 (2018).

14. Gendron, T.F., et al. Antisense transcripts of the expanded C9ORF72 hexanucleotide repeat form nuclear RNA foci and undergo repeat-associated non-ATG translation in c9FTD/ALS. Acta Neuropathol 126, 829844 (2013).

15. Ash, P.E., et al. Unconventional translation of C9ORF72 GGGGCC expansion generates insoluble polypeptides specific to c9FTD/ALS. Neuron 77, 639-646 (2013).

16. Mori, K., et al. The C9orf72 GGGGCC repeat is translated into aggregating dipeptide-repeat proteins in FTLD/ALS. Science 339, 1335-1338 (2013).

17. Zu, T., et al. RAN proteins and RNA foci from antisense transcripts in C9ORF72 ALS and frontotemporal dementia. Proc Natl Acad Sci U S A 110, E4968-4977 (2013).

18. May, S., et al. C9orf72 FTLD/ALS-associated Gly-Ala dipeptide repeat proteins cause neuronal toxicity and Unc119 sequestration. Acta Neuropathol 128, 485-503 (2014).

19. Mizielinska, S., et al. C9orf72 repeat expansions cause neurodegeneration in Drosophila through argininerich proteins. Science 345, 1192-1194 (2014).

20. Tao, Z., et al. Nucleolar stress and impaired stress granule formation contribute to C9orf72 RAN translation-induced cytotoxicity. Hum Mol Genet (2015).

21. Zhang, Y.J., et al. Aggregation-prone c9FTD/ALS poly(GA) RAN-translated proteins cause neurotoxicity by inducing ER stress. Acta Neuropathol 128, 505-524 (2014).

22. Freibaum, B.D., et al. GGGGCC repeat expansion in C9orf72 compromises nucleocytoplasmic transport. Nature 525, 129-133 (2015).

23. Loveland, A.B., et al. Ribosome inhibition by C9orf72-ALS/FTD poly-PR and poly-GR proteins revealed by cryo-E $<$. BioRxiv (2020).

24. Bensimon, G., Lacomblez, L. \& Meininger, V. A controlled trial of riluzole in amyotrophic lateral sclerosis. ALS/Riluzole Study Group. N Engl J Med 330, 585-591 (1994).

25. Rothstein, J.D. Edaravone: A new drug approved for ALS. Cell 171, 725 (2017). 
26. Cirak, S., et al. Exon skipping and dystrophin restoration in patients with Duchenne muscular dystrophy after systemic phosphorodiamidate morpholino oligomer treatment: an open-label, phase 2, dose-escalation study. Lancet 378, 595-605 (2011).

27. Khvorova, A. \& Watts, J.K. The chemical evolution of oligonucleotide therapies of clinical utility. Nat Biotechnol 35, 238-248 (2017).

28. Crooke, S.T., Witztum, J.L., Bennett, C.F. \& Baker, B.F. RNA-Targeted Therapeutics. Cell Metab 27, $714-$ 739 (2018).

29. Jafar-Nejad, P., et al. The atlas of RNase H antisense oligonucleotide distribution and activity in the CNS of rodents and non-human primates following central administration. Nucleic Acids Res (2020).

30. Kordasiewicz, H.B., et al. Sustained therapeutic reversal of Huntington's disease by transient repression of huntingtin synthesis. Neuron 74, 1031-1044 (2012).

31. Tabrizi, S.J., Smith, A.V. \& Bennett, C.F. Targeting Huntingtin in Patients with Huntington's Disease. Reply. N Engl J Med 381, 1181-1182 (2019).

32. McCampbell, A., et al. Antisense oligonucleotides extend survival and reverse decrement in muscle response in ALS models. J Clin Invest 128, 3558-3567 (2018).

33. Miller, T., et al. Phase 1-2 Trial of Antisense Oligonucleotide Tofersen for SOD1 ALS. N Engl J Med 383, 109-119 (2020).

34. Peters, O.M., et al. Human C9ORF72 Hexanucleotide Expansion Reproduces RNA Foci and Dipeptide Repeat Proteins but Not Neurodegeneration in BAC Transgenic Mice. Neuron 88, 902-909 (2015).

35. O'Rourke, J.G., et al. C9orf72 BAC Transgenic Mice Display Typical Pathologic Features of ALS/FTD. Neuron 88, 892-901 (2015).

36. Donnelly, C.J., et al. RNA toxicity from the ALS/FTD C9ORF72 expansion is mitigated by antisense intervention. Neuron 80, 415-428 (2013).

37. Lagier-Tourenne, C., et al. Targeted degradation of sense and antisense C9orf72 RNA foci as therapy for ALS and frontotemporal degeneration. Proc Natl Acad Sci U S A 110, E4530-4539 (2013).

38. Krieg, A.M. CpG motifs in bacterial DNA and their immune effects. Annu Rev Immunol 20, 709-760 (2002).

39. Almeida, S., et al. Modeling key pathological features of frontotemporal dementia with C9ORF72 repeat expansion in iPSC-derived human neurons. Acta Neuropathol 126, 385-399 (2013).

40. Mizielinska, S., et al. C9orf72 frontotemporal lobar degeneration is characterised by frequent neuronal sense and antisense RNA foci. Acta Neuropathol 126, 845-857 (2013).

41. Sareen, D., et al. Targeting RNA foci in iPSC-derived motor neurons from ALS patients with a C9ORF72 repeat expansion. Sci Transl Med 5, 208ra149 (2013).

42. Rigo, F., et al. Pharmacology of a central nervous system delivered 2'-O-methoxyethyl-modified survival of motor neuron splicing oligonucleotide in mice and nonhuman primates. J Pharmacol Exp Ther 350, 4655 (2014).

43. Geary, R.S., et al. Effect of dose and plasma concentration on liver uptake and pharmacologic activity of a 2'-methoxyethyl modified chimeric antisense oligonucleotide targeting PTEN. Biochem Pharmacol 78, 284-291 (2009).

44. Eckstein, F. Phosphorothioates, essential components of therapeutic oligonucleotides. Nucleic Acid Ther 24, 374-387 (2014).

45. Agrawal, S. Importance of nucleotide sequence and chemical modifications of antisense oligonucleotides. Biochim Biophys Acta 1489, 53-68 (1999).

46. Whitesell, L., et al. Stability, clearance, and disposition of intraventricularly administered oligodeoxynucleotides: implications for therapeutic application within the central nervous system. Proc Natl Acad Sci U S A 90, 4665-4669 (1993).

47. Campbell, J.M., Bacon, T.A. \& Wickstrom, E. Oligodeoxynucleoside phosphorothioate stability in subcellular extracts, culture media, sera and cerebrospinal fluid. J Biochem Biophys Methods 20, 259-267 (1990).

48. Sztainberg, Y., et al. Reversal of phenotypes in MECP2 duplication mice using genetic rescue or antisense oligonucleotides. Nature 528, 123-126 (2015).

49. Becker, L.A., et al. Therapeutic reduction of ataxin-2 extends lifespan and reduces pathology in TDP-43 mice. Nature 544, 367-371 (2017).

50. Tabrizi, S.J., et al. Targeting Huntingtin Expression in Patients with Huntington's Disease. N Engl J Med 380, 2307-2316 (2019). 
51. Moazami, M.P., et al. Quantifying and mitigating motor phenotypes induced by antisense oligonucleotides in the central nervous system. BioRxiv (2021).

52. DeJesus-Hernandez, M., et al. Novel p.Ile151Val mutation in VCP in a patient of African American descent with sporadic ALS. Neurology 77, 1102-1103 (2011).

53. Wen, X., et al. Antisense proline-arginine RAN dipeptides linked to C9ORF72-ALS/FTD form toxic nuclear aggregates that initiate in vitro and in vivo neuronal death. Neuron 84, 1213-1225 (2014).

54. Tran, H., et al. Differential Toxicity of Nuclear RNA Foci versus Dipeptide Repeat Proteins in a Drosophila Model of C9ORF72 FTD/ALS. Neuron 87, 1207-1214 (2015).

55. Jovicic, A., et al. Modifiers of C9orf72 dipeptide repeat toxicity connect nucleocytoplasmic transport defects to FTD/ALS. Nat Neurosci 18, 1226-1229 (2015).

56. Zhang, Y.J., et al. Poly(GR) impairs protein translation and stress granule dynamics in C9orf72-associated frontotemporal dementia and amyotrophic lateral sclerosis. Nat Med 24, 1136-1142 (2018).

57. Suzuki, H., Shibagaki, Y., Hattori, S. \& Matsuoka, M. The proline-arginine repeat protein linked to C9ALS/FTD causes neuronal toxicity by inhibiting the DEAD-box RNA helicase-mediated ribosome biogenesis. Cell Death Dis 9, 975 (2018).

58. Yuva-Aydemir, Y., Almeida, S., Krishnan, G., Gendron, T.F. \& Gao, F.B. Transcription elongation factor AFF2/FMR2 regulates expression of expanded GGGGCC repeat-containing C9ORF72 allele in ALS/FTD. Nat Commun 10, 5466 (2019).

59. Moens, T.G., et al. C9orf72 arginine-rich dipeptide proteins interact with ribosomal proteins in vivo to induce a toxic translational arrest that is rescued by eIF1A. Acta Neuropathol 137, 487-500 (2019).

60. Coyne, A.N., et al. G4C2 Repeat RNA Initiates a POM121-Mediated Reduction in Specific Nucleoporins in C9orf72 ALS/FTD. Neuron (2020).

61. Kumar, R., et al. The first analogues of LNA (locked nucleic acids): phosphorothioate-LNA and 2'-thioLNA. Bioorg Med Chem Lett 8, 2219-2222 (1998).

62. DeVos, S.L. \& Miller, T.M. Direct intraventricular delivery of drugs to the rodent central nervous system. $J$ Vis Exp, e50326 (2013). 


\section{Figures}

a

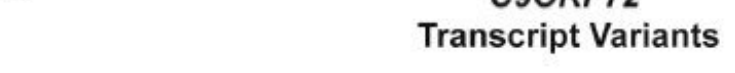

\section{Protein Isoforms}

Short Isoform 222 aa (25kDa)
V3

V2

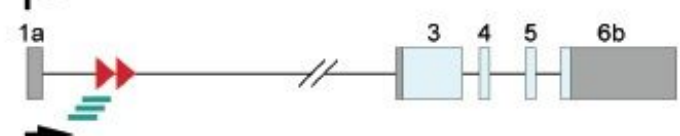

3
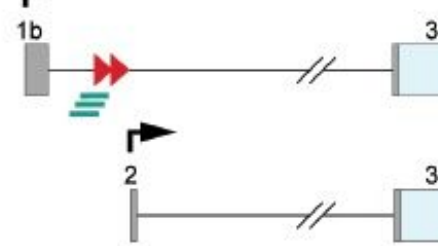
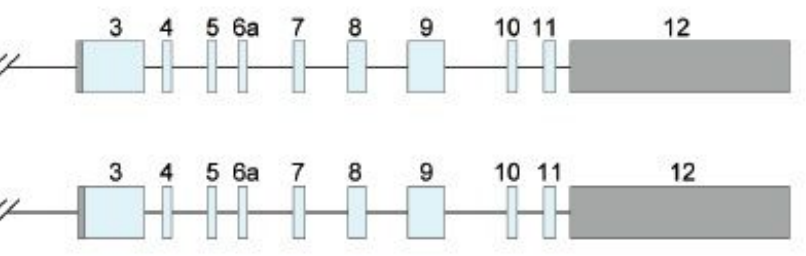

$\|$ UTR $\square$ Exons $\ggg \mathrm{G}_{4} \mathrm{C}_{2}$ Repeat $\quad$ ASO

b

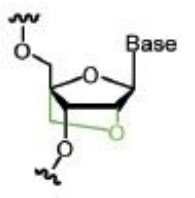

LNA

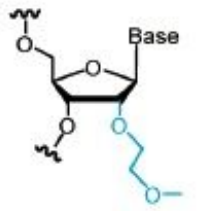

2'-O-MOE RNA $\triangle \mathrm{PS}$

O DNA

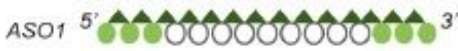

aso ${ }^{2}$ A A

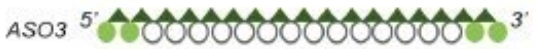

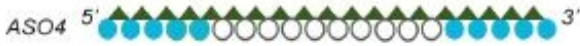

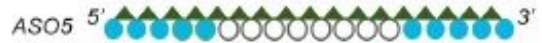

C Patient Derived Fibroblasts

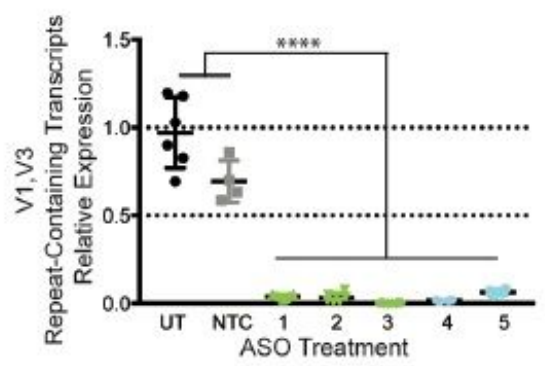

f

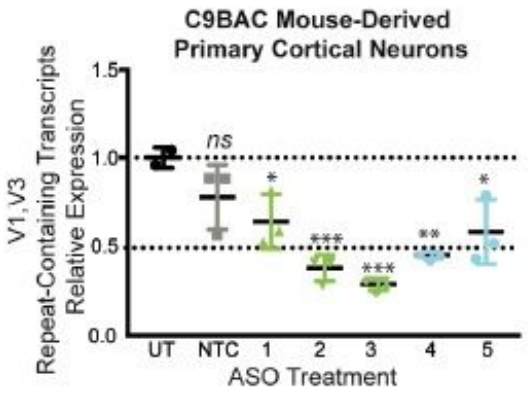

Figure 1

G4C2 targeting LNA and MOE modified ASO reduces the C9ORF72 repeat containing transcripts in patient derived fibroblasts and C9BAC mouse derived neurons. (a) Schematic of C9ORF72 transcript variants and of the two protein isoforms as named in PubMed. The repeat expansion (red triangles) located in the first intron is expressed in variants 1 and 3 (V1-V3). Variant 2 (V2), the most abundant, starts form a different transcription start site (black arrow) and does not include the repeat expansion. V2 and V3 encode the main C9ORF72 protein isoform. Grey boxes represent untranslated regions (UTR); light blue boxes, exons; black lines, introns. ASOs used in this study (green bars) target the intronic region flanking the repeat expansion. (b) Schematic of ASOs and their chemical modifications used in this study: green circle, 
locked nucleic acid (LNA); blue circle, 2'-0-2-methoxyethyl RNA (2'-O-MOE); empty circle, DNA; dark green triangle, phosphorothioate nucleotide linkage (PS). (c) LNA and 2'-OMOE ASOs significantly reduce the level of repeat containing transcripts at a dose of $100 \mathrm{nM} 72 \mathrm{hrs}$ after lipid-mediated delivery in patient derived fibroblasts as measured by qRT-PCR. (d) Representative images of RNA foci visualized by FISH in patient derived fibroblasts untreated or treated with ASO for $72 \mathrm{hrs}$. (e) \% of nuclei without foci (light grey), with 1-4 foci (dark grey) or with more than 5 foci (black) when cells were treated with no ASO, a nontargeting control ASO (NTC), LNA modified ASOs (ASOs 1-3) or 2'-O-MOE modified ASOs (ASOs 4-5); 50 cells examined for each group. (f) LNA and 2'-O-MOE ASOs significantly reduce the level of repeatcontaining transcripts after two weeks treatment with $1 \mu \mathrm{M}$ ASO without lipid assistance (gymnotic delivery) in primary cortical neurons derived from C9BAC mice.

a

\begin{tabular}{llc}
\hline Name & Sequence (5' - - $^{\prime}$ ) & $\begin{array}{c}\text { Acute Toxicity } \\
\text { (WT mice) }\end{array}$ \\
\hline ASO1 & CCCTAGCGCGCGCGACT & High \\
ASO2 & CCCGGCCCCTAGCGCGCGAC & High \\
\hline ASO3 & GCCCCTAGCGCGCGACTC & Low \\
\hline ASO4 & CCCGGCCCCTAGCGCGCGAC & High \\
\hline ASO5 & GCCCCTAGCGCGCGACTC & Low \\
\hline
\end{tabular}

b

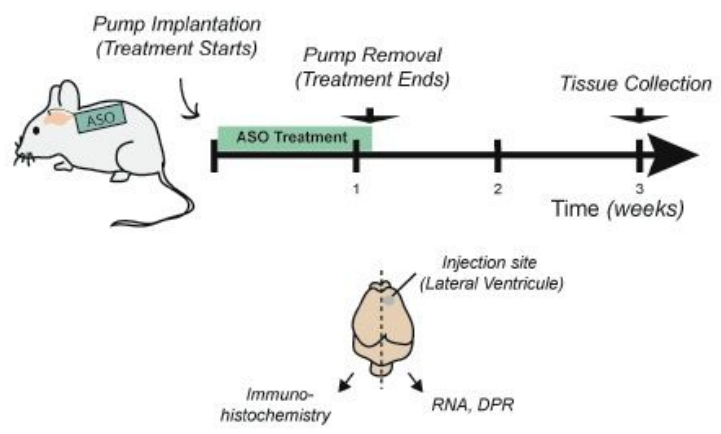

C

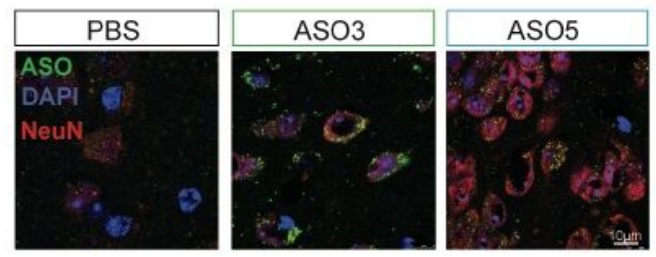

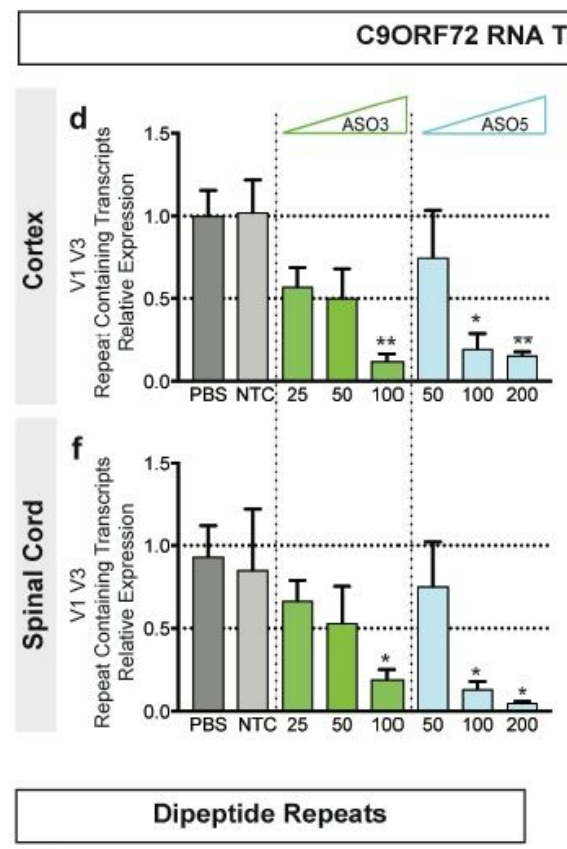

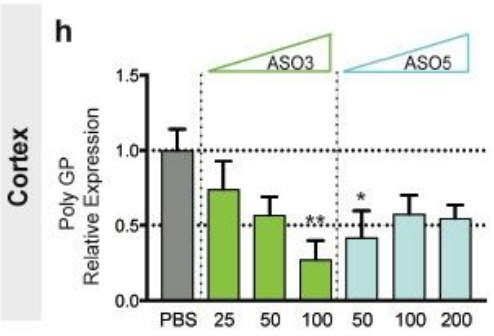

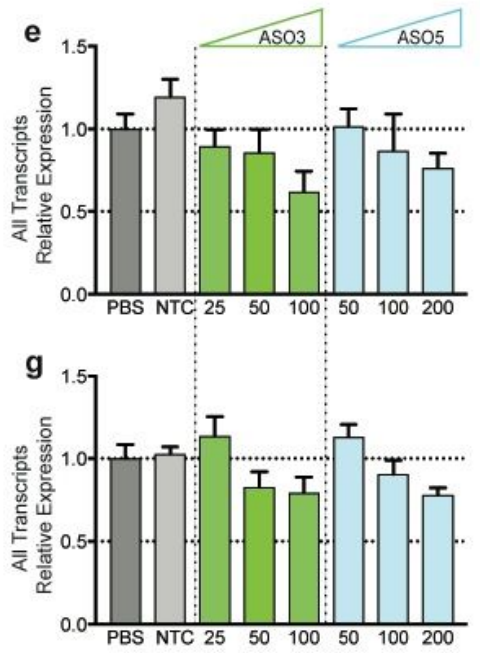
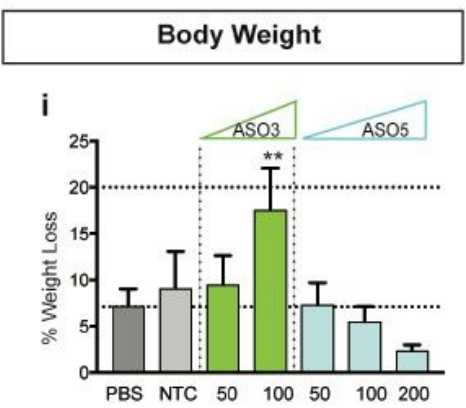

\section{Figure 2}

G4C2 targeting LNA and MOE modified ASOs selectively reduces the C9ORF72 repeat containing transcripts and peptides after CNS infusion in C9BAC mice (a) ASO sequences and their in vivo response in 4-6-month-old wild-type mice after ICV bolus injection: bold green, LNA; bold blue, MOE; italic black, DNA. (b) Schematic of experimental design in heterozygous C9BAC mice. Vehicle control (PBS), a nontargeting control ASO (NTC) and 25 to $200 \mathrm{nmol}$ of ASO3 and ASO5 were infused into the right lateral ventricle of 5-6-monthold C9BAC mice over 10 days. Brain and spinal cord were harvested and dissected two weeks after pump removal for RNA and DPR analysis (spinal cord and ipsilateral brain hemisphere), 
and ASO staining (contralateral brain hemisphere). (c) ASO3 and ASO5 (green) are taken up in neurons (red) - nuclei counterstaining in blue. (d-g) Expression of V1-V3 repeat containing transcripts $(\mathrm{d}, \mathrm{f})$ and all transcripts $(e, g)$ in cortex and spinal cord quantified by qRT-PCR in mice infused with PBS (dark grey), NTC ASO (light grey), ASO3 (green) or ASO5 (blue) at the indicated dose. For each dose level, n= 5-7, except NTC group $(n=3)$. (h) Relative expression of polyGP in the cortex of mice treated with ASO3 (green) and ASO5 (blue) assayed by sandwich immunoassay. Data are represented as mean \pm SEM. (i) \% of body weight loss at end point relative to before treatment.
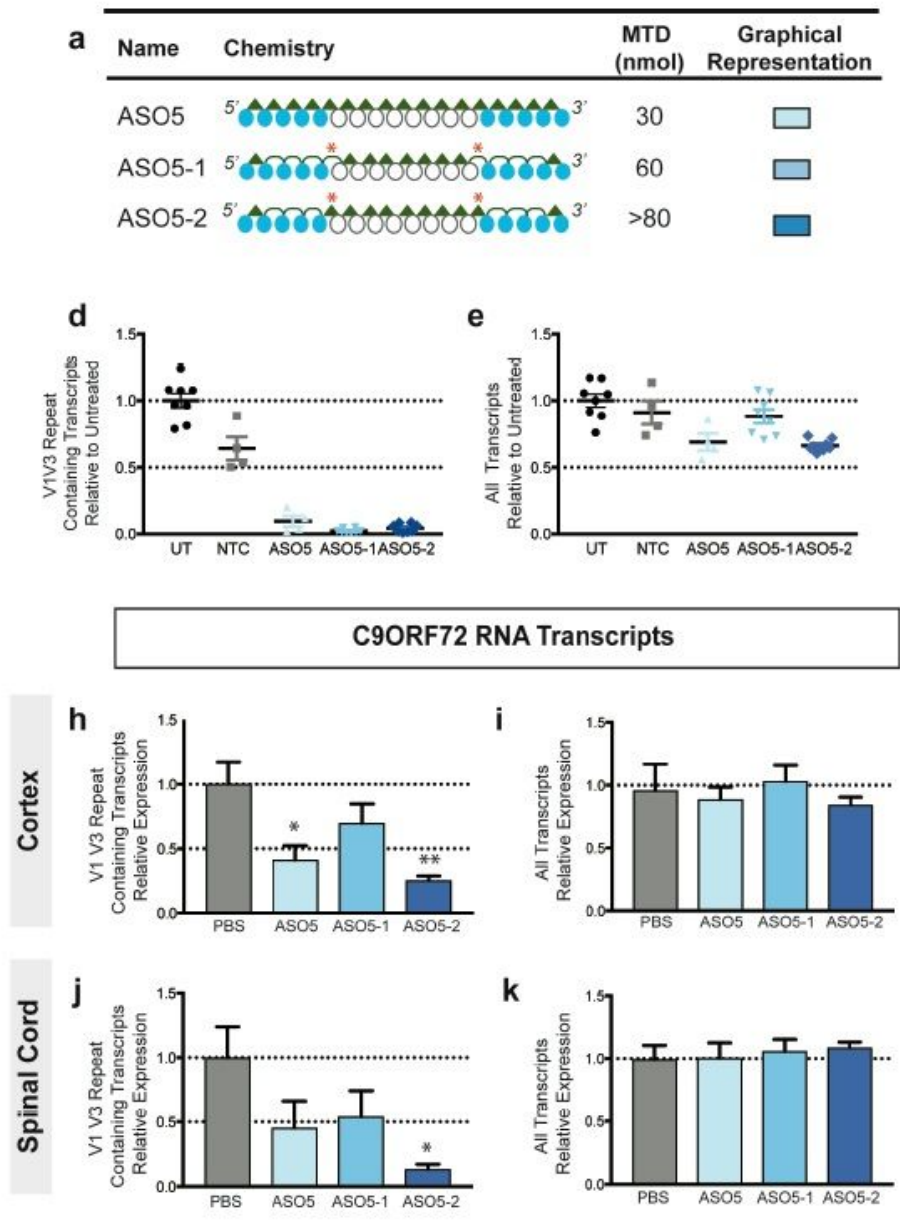
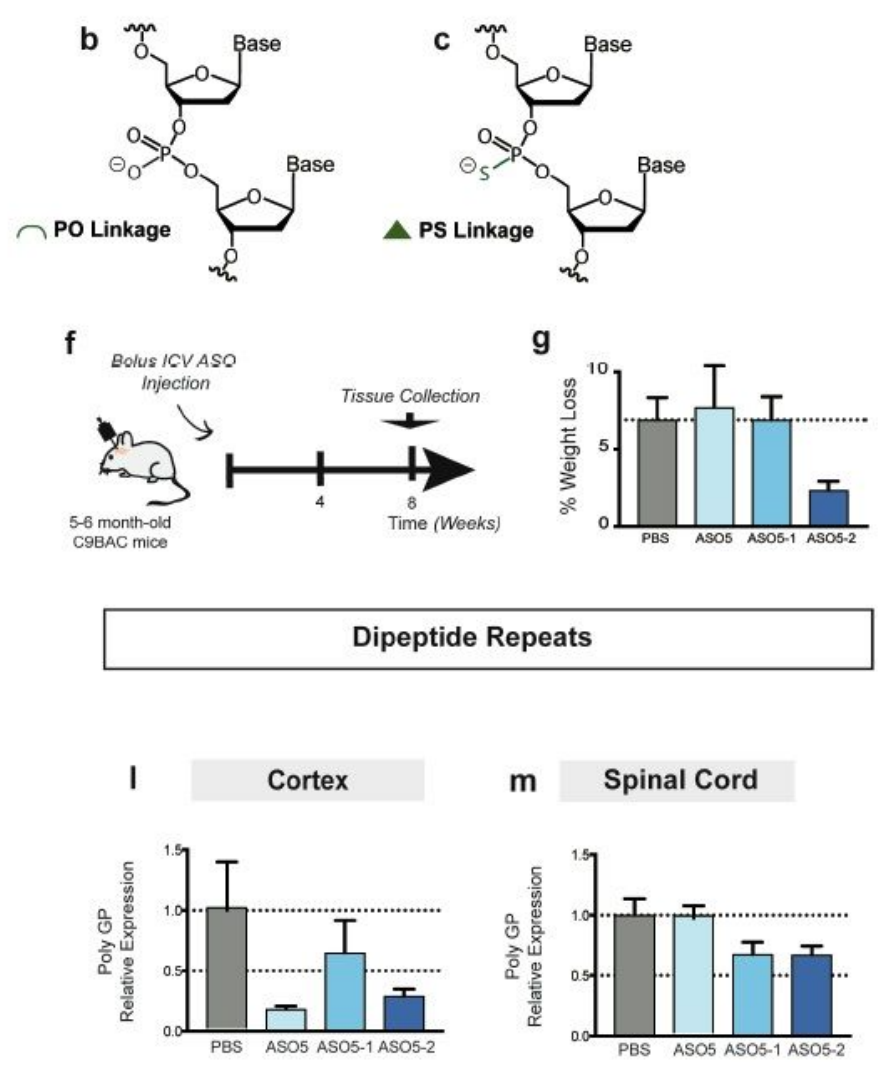

\section{Figure 3}

Reducing the phosphorothioate content of ASO5 improves its safety profile without reducing its biological activity after CNS administration in C9BAC mice (a) Chemistry and maximum tolerated dose (MTD) in nmol of ASO5 and its mixed-backbone derivatives (ASO5-1 and AS05-2). Maximum Tolerated Doses (MTD) were evaluated in vivo in 5-6-month-old wild-type C57BI/ 6 mice for up to 8 weeks after ICV bolus injections. (a-b) green hemi-circle, phosphodiester linkage (PO); (a-c) green triangle, phosphorothioate linkage (PS). In (a) closed blue circle, 2'-O-MOE; open circle, DNA: red star highlights the position of PS/PO difference between AS05-1 and ASO5-2. (d-e) Effect of 100nM of a mixed PS/PO on ASO5 efficacy in patient-derived fibroblasts on expression of V1,V3 repeat-containing transcripts (d) or all transcripts (e). (f) Schematic of experimental design in heterozygous C9BAC mice. Vehicle control (PBS), 
ASO5, AS05-1 or ASO5-2 were injected into the right lateral ventricle of 5-6 month old C9BAC mice. Brain and spinal cord were harvested and dissected eight weeks after treatment for RNA and DPR analysis. (g) No significant change in body weight was observed eight weeks after treatment when mice were treated with ASO. (h-m) Expression of V1-V3 repeat containing transcripts $(h, j)$ and all transcripts $(i, k)$ in cortex and spinal cord quantified by qRTPCR and polyGP $(\mathrm{l}, \mathrm{m})$ in mice treated with PBS (dark grey), ASO5 (light blue), ASO5-1 (medium blue) and ASO5-2 (dark blue) eight weeks after administration of 30nmol of each ASO. For each ASO group, $n=5-7$.
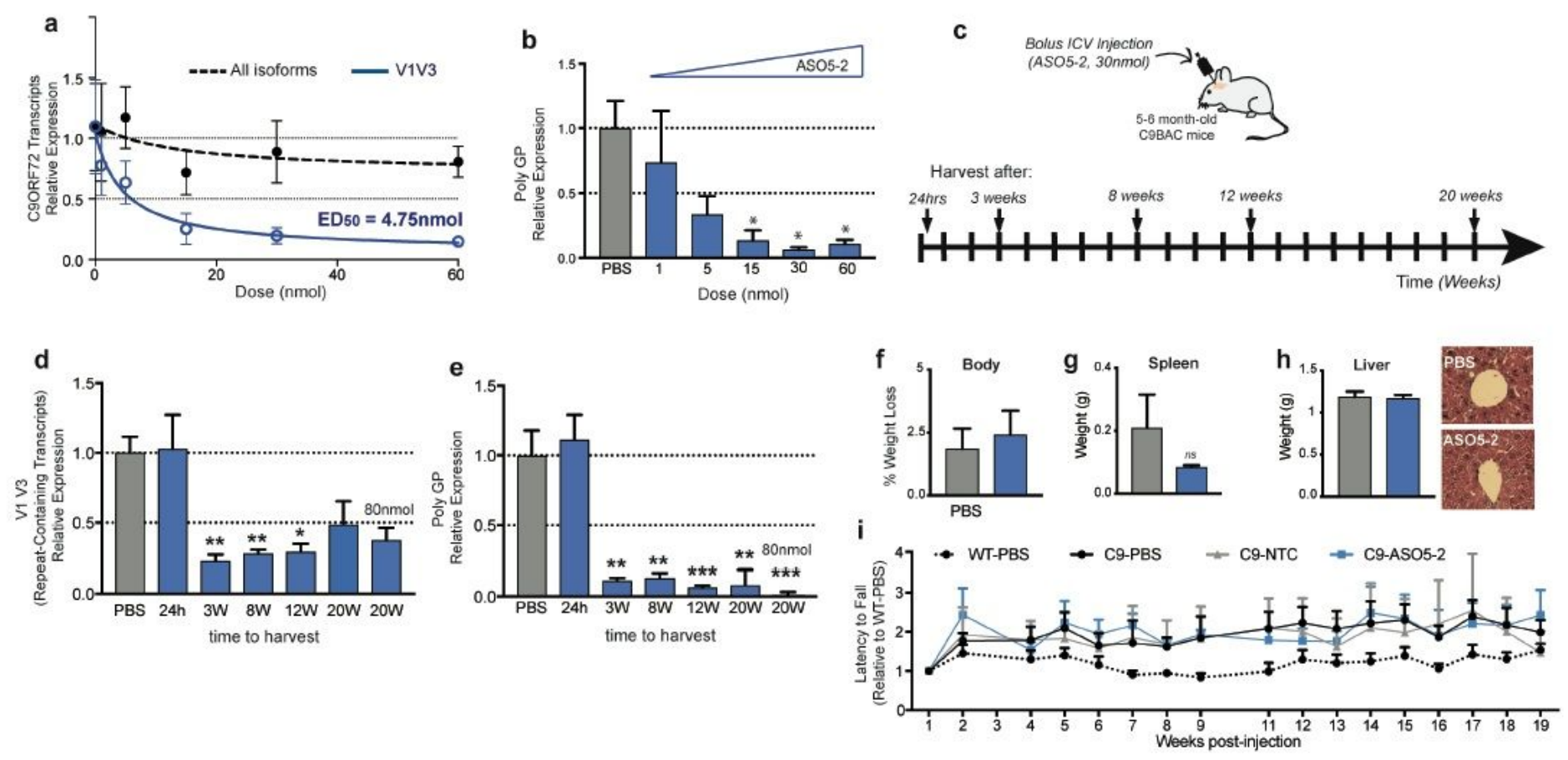

\section{Figure 4}

Sustained and potent effect of ASO5-2 in the CNS of C9BAC mice. (a-b) Dose-dependence of gene silencing by ASO5-2 in heterozygous C9BAC mice. Vehicle control (PBS) and a single injection of 1, 5, 15, 30 , or $60 \mathrm{nmol}$ of ASO5-2 was administered into the lateral ventricle of 5-6-month-old heterozygous C9BAC mice. Expression of V1-V3 repeatcontaining transcripts and all transcripts (a) and polyGP (b) was analyzed 3 weeks after injection. (c-i) A time course experiment was performed in heterozygous C9BAC mice treated with $30 \mathrm{nmol}$ of ASO5-2, tissues were collected and analyzed at $24 \mathrm{~h}$ or $3,8,12$ and 20 weeks after treatment (schematic shown in panel c); an $80 \mathrm{nmol}$ dose was also harvested at 20 weeks only. Expression of $\mathrm{V} 1, \mathrm{~V} 3$ repeats containing transcripts (d) and polyGP (e) was analyzed in cortex $24 \mathrm{~h}$ or 3,8 , 12 or 20 weeks after a single dose injection of AS05-2. No significant change in body weight (f), spleen (g) liver (h) and kidney (not shown) weight or morphology was observed after treatment with ASO5-2 (blue) relative to PBS (grey) 8 weeks after treatment. ASO treatment did not alter motor performance on a 5-minute accelerating rotarod task during the treatment course (i). For each treatment or dose group, $n=6$ 7. 


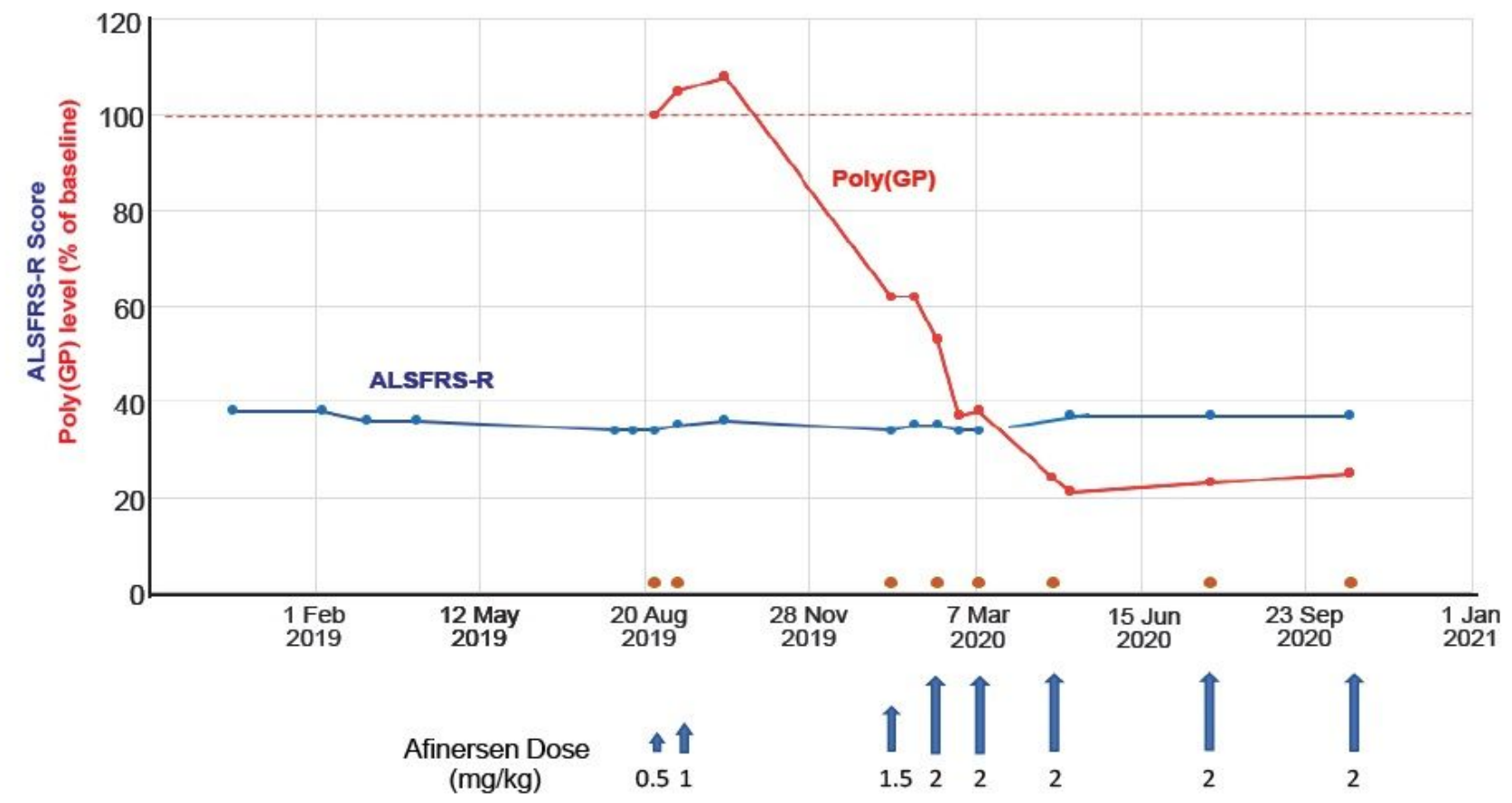

Figure 5

Clinical summary and afinersen (ASO5-2) dosing. A single patient received multiple doses of ASO5-2 as shown below the graph. The patient's ALSFRS-R score before and during treatment is shown as a blue line and points. The patient's poly(GP) DPR level, relative to the baseline $(100 \%)$ is shown as a red line and points.

\section{Supplementary Files}

This is a list of supplementary files associated with this preprint. Click to download.

- SuppInfoC9ASONatMed05Feb2021.pdf 